\title{
A Comparison of Software Tools for Occupational Biomechanics and Ergonomic Research
}

\author{
Pamela McCauley Bush", Susan Gaines, \\ Fatina Gammoh and Shanon Wooden \\ Ergonomics Laboratory, \\ Department of Industrial Engineering and Management Systems, \\ University of Central Florida, Orlando, FL
}

USA

\section{Introduction}

The purpose of this study was to evaluate and compare commercially available software tools in ergonomics and biomechanics research. The project provides a survey of select biomechanical software tools and also gives a detailed analysis of two specialized packages, 3DSSPP and JACK as well as examples of applications where one or the other may be better suited. A summarized comparison of these two packages is provided.

Three research projects in the Ergonomics Laboratory at the University of Central Florida were used to evaluate the software tools in this study. This study, entitled A Human-Centered Assessment of Physical Tasks of First Responders in High Consequence Disasters, looks at three of the physical tasks associated with first responders in disaster management (i.e., emergency management and response). The third case is a preliminary study to analyze the biomechanics associated with interactive gaming. The associated output not only provides a direct comparison of the two software tools, but also provides recommendations for the preferred simulation tool for appropriate biomechanical analysis for each of the three projects. The results identify the physical tasks which may place subjects at risk of physical injury and possible cumulative trauma disorders (i.e. work related musculoskeletal disorders (WMSD)). The results of the simulation analysis can be useful to researchers in assessing risks, developing worker training, selecting appropriate personal protective equipment, and recommending ergonomic interventions to mitigate risks.

For each of the three research projects being evaluated, select task elements were identified for evaluation. The task elements (or activities) selected represent a cross-section of typical physical tasks performance in physically intensive task performance (i.e. load lifting, carrying weight, or awkward posture). These tasks were simulated from photographs taken during actual task performance or still photos taken from videos. For the solid waste

${ }^{*}$ Corresponding Author 
collection project, the worker was observed lifting and emptying a full canister, and moving an empty canister back into place. For the disaster management research, three tasks commonly associated with first responders were evaluated including victim extraction, supply distribution, and moving the injured. The tasks were simulated by positioning virtual models in the same postures as workers and estimating the loads. Variables which were considered included uneven ground in which workers must work, lifting loads, and body and limb postures. Variables which could not be simulated using the software tools included temperature, humidity, physical fatigue, mental stress, and chemical, biological and environmental hazards. The interactive gaming project involved observing a subject playing a controller-free video game and simulating some of the postures that were commonly performed during game play.

Software developed by the University of Michigan, 3DSSPP, was used to assess tasks from all three research projects. The 3DSSPP results were used to evaluate the loads, balance and stresses on the virtual humans. The same tasks were evaluated with the JACK software, developed by Siemens Corporation. The summary reports generated by each of the software tools were compared and analyzed for each project.

\section{History and significance}

The comparison of software tools for biomechanical analysis is an important aspect for understanding the most applicable tools for a given research project. In a review of the literature, few studies were identified that performed an analysis of the different features of comparable biomechanical modeling software. The growth of computer based analysis tools, dictates a need for the unbiased research community to provide analyses that can offer objective feedback on the use of these analysis tools.

Three environments that contain potentially hazardous postures in ergonomics and biomechanics were identified. These three projects are ongoing research efforts in the Ergonomics Laboratory at the University of Central Florida and provided an opportunity for a comparative study of related software products. Below are brief summaries of these projects.

\subsection{Ergonomic study in solid waste collection}

Municipal Solid Waste collection is a necessary activity all around the world and is associated with occupational injuries due to ergonomic risk factors including lifting, heavy load handling, awkward postures, long task durations and high levels of repetition. In the past, waste has been collected manually from customers, and has often resulted in frequent injuries to the workers. Technological development has introduced automated and semiautomated collection systems that, according to manufacturer's claims, enhance worker safety, collection productivity while at the same time reducing workers compensation claims. Thus, such advantages should balance increases in equipment cost; however, some experts suggest that for automated and semi-automated waste collection systems the capital, operating and maintenance costs are higher than costs associated with manual collection.

From the published literature, it was noticed that relatively little research has been published on ergonomics and safety in the manual waste collection industry. Additionally, 
the field is lacking a comprehensive study that assesses and compares the ergonomic and biomechanics issues associated with waste collection at varying levels of automation including manual, semi-automatic and automatic. This study will fill the research gap by providing an ergonomic and biomechanics assessment of the three primary approaches to waste collection.

The study utilized observational analysis, laboratory analysis and a review of historical data, where surveys were conducted for solid waste collectors and safety personnel of different waste companies in Orlando, Florida to understand the factors affecting waste collectors' safety. The focus will be on the type of waste collection tasks performed in residential communities,

Ergonomics and biomechanics evaluation techniques included postural analysis, lifting analysis, assessment of musculoskeletal risk and holistic assessment of occupational risks for workers at all three levels of automation. A detailed review of the injury data collected by the U.S. Bureau of Labor Statistics (BLS) was performed to evaluate the nature and frequency of injury incidents over time in solid waste collection field. The study will establish a foundation for additional research and recommendations for mitigating risks at all levels of task performance.

\subsection{A human-centered assessment of physical tasks of first responders in high consequence disasters}

This human-centered study is the initial step in developing a methodology to categorize and analyze physical tasks performed by first responders in high consequence disasters from a human factors' and biomechanics perspective. Four key phases of Disaster Management include preparedness, response, recovery, and mitigation. The tasks analyzed in this study occur in the response phase. The software tools, 3DSSPP and JACK, allowed evaluation of biomechanical risks associated with the tasks performed $\mathrm{n}$ the response phase. For comparative purposes the physical tasks evaluated can be partitioned into three categories: 1) Victim extraction, 2) Moving of injured, and 3) Distribution of supplies (food, water, or temporary housing supplies). Photographs of emergency workers and volunteers fulfilling these roles were retrieved from past disasters and subject matter experts. Additionally, task activities and related postures and load handling was simulated in the software environment.

The volume of rescue workers in a high consequence disaster is difficult to quantify. While professionally trained rescuers such as firemen and policemen will provide aid, according to Kano, Siegel and Bourque (2005), "It is (also) recognized that members of the lay public are often the actual 'first responders' in many disaster events." Issues related to mental stress of witnessing widespread death and devastation have been widely researched with regard to first responders. As a result of the World Trade Center Disaster in 2001, first responder health problems related to pulmonary issues due to ingested dust and particles at disaster site have also been well-documented. Personal protective equipment (PPE) is endorsed by the Occupational Safety and Health Administration (OSHA) based on data from previous disasters; however, recommended PPE equipment tends to be in response to environmental, biological or chemical risks. However specific biomechanical risks have not been widely studied among responders in high consequence emergency response. 
Therefore, potentially valuable technology and personal protective equipment (PPE) such as lifting aids or back belts used in lifting tasks are missing from disaster PPE recommendations. The frequency of weather-related disasters has increased in the past ten years. From 1980 through 2009, there have been 96 weather-related disasters in which overall damages reached or exceeded $\$ 1$ billion per event (NCDC). Scientists theorize the increase is related to global warming. Whatever the cause, it is clear that the frequency with which disaster workers and volunteers will need to provide aid will continue to increase. The lack of training and literature with regard to mitigation of risks to first responders as related to physical tasks, points to the need for more research in this area.

Research has focused on mental health risks such at Post-Traumatic Stress Disorder (PTSD), environmental risks such as chemicals, electrical risks due to downed power lines, and biological hazards which include "insect bites/stings, mammal/snake bites, and exposure to molds and other biological contaminants as a result of water damage, and sewage infiltration in low-lying areas". (Stull, 2006) Despite the lack of research and literature regarding risks and injuries of first responders as a result of the physical tasks performed, back injuries account for $31 \%$ of all workers' compensation claims in the United States. This fact alone indicates the need to study rescue worker safety with regard to the physical tasks performed and subsequent risks incurred by carrying out these tasks.

If physical tasks can be categorized and evaluated for risk utilizing software tools for simulation, researchers can identify those tasks which place rescue workers at greatest risk. Once these tasks are identified, collection of real-time data from disaster sites can be collected and analyzed. These results can be compared and validated by recreation of the tasks in a laboratory setting and analysis with the software simulation tools. Action in the form of enhancements to training and additional PPE recommendations can be taken to reduce the risks to these workers. Ultimately, both victims and responders will benefit from having a healthier work force that can provide faster and more efficient response, further preserving lives and expediting rescues.

\subsection{Biomechanical assessment of postures associated with Interactive gaming}

The advent of movement and gesture-based video gaming systems such as the Nintendo Wii, Playstation Move and Xbox Kinect have recently taken the world of gaming and computer interaction to a whole new level. Rather than controlling the game with one's digits, the player's entire body can be used to control his or her actions within the gaming interface. This sort of technology introduces a new level of activity to users who were once glued to their seats during play. Conversely, this technology has raised concerns about injuries due to the overuse of the motion-controlled mode of entertainment.

The Wii system is the first of the motion-based games on the market, as it was released in 2006. Although the technology is relatively new, there have been reports of Wii-related injuries in medical literature (Collins, 2008). Injuries that were once considered athleticrelated are now occurring in individuals who play in virtual games environments. This phenomenon is more likely to occur in a sedentary population participating in the activity (Barron, 2008). One study documents an emergency surgery that was performed on a 16 year-old boy for a Lateral patella dislocation; another serious case involved a 23-year-old woman who suffered a Meniscus tear as a result of playing 10-pin bowling on a Nintendo 
Wii video game. Based on interviews with orthopedists and sports medicine physicians, the majority of Kinect-related injuries are not severe. Some cases that doctors have seen range from twisted knees, sprained ankles, strains, swelling, and some repetitive stress problems (Das, 2009). Patients complaining of injuries range from young children, to teens, to young adults, and even elderly adults. A common issue with these injuries is that players do not realize that full force and motion is not required for the game to acknowledge the action. Instead of a minuscule jump, the user might perform a full leap. If a swing action is required, many users may force a full swing when the game may only require a flick of the wrist. Some medical professionals suggest that sports injuries and cumulative trauma disorders may be likely directions for the types of injuries that may occur due to interactive game use (Barron, 2008).

Presently, interactive gaming technology is new and little published scientific research exists, particularly in the area of biomechanics. However, this poses an excellent opportunity to identify the possible risks associated with the use and over use of the systems. Performing ergonomic and biomechanical evaluations of these motion-activated games could benefit customers, manufacturers, and medical professionals, alike.

The objective of this study is to employ human modeling and simulation tools to identify potential hazards associated with some of the awkward postures exhibited during game play. The 3DSSPP and JACK programs are mainly used in analyzing occupational manual material-handling tasks. In this study these software tools will be used to simulate postures of the subject to determine if these products can go beyond occupational applications to support healthy biomechanics in design of a recreational product such as an interactive game.

\section{Literature review}

An internet search of software available for biomechanical analysis resulted in a significant number of options. The majority of the software offered online tends toward biomechanical evaluation for sports applications. Several of the software packages claim to accept userprovided video for analysis, but demonstrations of the software have established that these tools cannot readily accept typical user video. The videos to be used as input for most of the software must be made at a particular resolution, recorded from certain angles or be in a format which requires special, sometimes expensive, hardware. The general survey of biomechanical analysis software reveled that there are three main categories of software: $2 \mathrm{D}$ Video Analysis, 3D Motion Capture Analysis, and Human Modeling and Simulation programs. Several of these packages are discussed below.

\subsection{D video analysis}

\subsubsection{MotionView}

MotionView video analysis software for sports is video coaching software that advertises it can accept input from any video camera and computer to analyze or coach sports and motion; however, the makers require the user to purchase special equipment from them to capture the videos. This software is used primarily for sports evaluation. "MotionView video analysis software for sports delivers features typically found in video analysis and 
swing analysis software costing much more. MotionView video analysis software for sports is golf swing analysis software, bowling video analysis software, and tennis stroke video analysis software! Improve any athletic skill with our video analysis software." The MotionSuite complete package costs $\$ 1180$ http:/ / www.allsportsystems.com/

\subsubsection{ProAnalyst professional}

ProAnalyst software initially seemed to be a promising tool in which user-supplied video could be downloaded and analyzed. While it does accept some user videos, there are restrictions with regard to the quality of resolution and the camera angles from which the video can be taken (side views only). Again, ProAnalyst ${ }^{\circledR}$ is used primarily for evaluation of sports; however, there are applications in the aerospace industry, such as tracking missile paths and speeds. ProAnalyst advertises that it "is the world's premier software package for automatically measuring moving objects with video. ProAnalyst allows you to import virtually any video and quickly extract and quantify motion within that video. Used extensively by NASA, engineers, broadcasters, researchers and athletes, ProAnalyst is the ideal companion software to any consumer, scientific and industrial video camera, and vice versa. With ProAnalyst, any video camera becomes a non-contact test instrument. ProAnalyst allows users to measure and track velocity, position, size, acceleration, location and other characteristics." ProAnalyst does provide the ability to export data into graphical formats, but it did not prove to be as user-friendly for occupational evaluation, and it required cameras which recorded at a higher resolution than the typical home video camera. The ProAnalyst Professional Edition, Ultimate Bundle costs \$9595. http://www.xcitex.com/html/proanalyst_applications_examples.php

\subsubsection{MaxTRAQ 2D}

MaxTRAQ 2D can use a standard camcorder to high speed camera for input. This program also features a manual or automatic digitizer that can be used to extract kinematic properties from standard AVI files. This feature is useful when markers cannot be placed on the subject. MaxTRAQ includes tools to measure distances, angles, center of mass, etc. The price of this software is $\$ 695$. http:// www.innovision-systems.com

\subsection{D motion capture}

\subsubsection{Visual3D professional}

C-Motion-Visual3D biomechanical analysis software is marketed as being "used for performance analysis and movement assessments." The applications appear to be more pertinent to the medical community. This software does require an existing motion capture system. For this system, cameras are not directly supported. Video data must be preprocessed into a digital format that Visual3D can process and analyze. Depending on the Motion capture setup, this may require additional software. This system also reports that data from Force Platform and EMG analog devices can be synchronized with the video. The cost for the Visual3D Professional (with Real-Time Biofeedback, Relational Database Export, Inverse Kinematics, 4-user License) is \$15995. http://www.cmotion.com/products/visual3d.php 


\subsubsection{MaxPRO}

MaxPRO is a motion caption and analysis product that can be used for research, clinical/Physical Therapy, biomechanics, sports, ergonomics, industrial/automotive, lab course/teaching. MaxPro offer 3D motion analysis without the use of a proprietary camera. This system can utilize standard camcorders to high speed, high resolution cameras. Some of the features of this program include up to a 32-camera configuration, tracking for up to 255 markers, video overlay, and graphs. The tools available can detect angles, velocity, and acceleration. This software price is listed as $\$ 4,995$. http:/ / www.innovision-systems.com

\subsubsection{SIMM}

SIMM Biomechanics Software Suite by MusculoGraphics "enables a detailed analysis, documentation and comparison of posture and movements"; however, it requires specialized software for simulation. It does not utilize video download features. http:/ / www.musculographics.com/

\subsubsection{ProAnalyst 3-D professional}

ProAnalyst 3-D Professional Edition uses video from two cameras to create a 3D analysis tool. The system requires a special calibration tool that allows for the user to "drag and drop two calibration images in the 3-D Manager window and let ProAnalyst automatically determine the positions of the cameras. Then, add analyzed videos and allow ProAnalyst to calculate where your tracked objects are in 3-dimensional space. Finally, export your data to a fully customizable 3 -axis plot and save a new video showing your analyzed event from any angle." The cost of this package is $\$ 14995$.

http://www.xcitex.com/html/proanalyst_applications_examples.php

\subsection{Modeling and simulation}

\subsubsection{DSSPP}

The 3DSSPP (3D Static Strength Prediction Program) was developed by The Center for Ergonomics at the University of Michigan College of Engineering. This program can be used in analyzing manual materials - handling tasks. Ergonomists, engineers, therapists and researchers, may use the software to evaluate and design jobs. This program allows for users to input anthropometric data, and obtain the forces and moments computed by the program, rather than by manual calculation. In addition, the program also combines the National Institute of Occupational Safety (NIOSH) lifting data and other additional reports to identify risks associated with a particular task. This software license costs \$1495 (University of Michigan).

\subsubsection{JACK}

JACK is a human simulation tool for populating designs with virtual people and performing human factors and ergonomic analysis. JACK is a human modeling and simulation tool. JACK, and its optional toolkits, provides human-centered design tools for performing ergonomic analysis of virtual products and virtual work environments. 
JACK enables you to size human models to match worker populations, as well as test designs for multiple factors, including injury risk, user comfort, reachability, line of sight, energy expenditure, fatigue limits and other important human parameters. This software license costs $\$ 2400$ www.siemens.com/tecnomatix

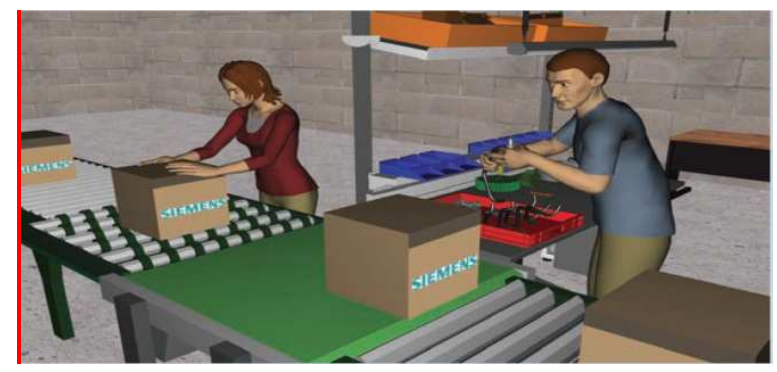

Fig. 1. Simulation from JACK software, Technomatix.

\subsubsection{Ergowatch}

Ergowatch is another computerized ergonomics package system. It consists of different ergonomic measurement tools that can help employers, ergonomists, and workers to estimate and interpret the physical loading associated with various jobs. The Ergowatch package provides the below tools for work evaluation:

1. The 4D Watbak Tool which is easy to use biomechanical modeling software, to calculate instantaneous and accumulated loads for the lower back and other major body joints, during various activities and to predict the relative risk of lower back injury

2. The NIOSH Tool which provides load limits for lifting and lowering activities (based on the 1981 and 1991 NIOSH Lifting Equations)

3. The Snook Tool: Provides load limits for lifting, lowering, pushing, pulling and carrying activities (based on the 1991 Revised Snook Tables)

4. The Physical Demands Description (PDD) Checklist Tool: Structures the description of physical movements and environmental conditions associated with a task group or job (adapted from the Ontario Ministry of Labor Physical Demands Analysis form). The cost of this package is $\$ 1500$. http://www.escs.uwaterloo.ca/brochure.pdf

\subsubsection{AnyBody modeling system}

The AnyBody Modeling System ${ }^{\mathrm{TM}}$ is a software system for simulating the mechanics of the live human body working in a particular environment. AnyBody has applications in the auto industry, medicine, the aerospace industry, sports analysis, research, and even defense. The software runs a simulation and calculates the associated mechanical properties including individual muscle forces, joint forces and moments, metabolism, elastic energy in tendons, antagonistic muscle actions and much more. AnyBody can also import data from Motion Capture systems. The pricing was not available without a full demonstration. The company is headquartered in Sweden. http:/ / www.anybodytech.com/index.php?id=26

A priority of this software research was to find tools which did not require a significant financial commitment, particularly with regard to specialized hardware, as that technology 
can be costly and often times the technology evolves quickly, rendering older generations of hardware obsolete. Due to the cost of software and required peripherals for motion analysis options, the next-best alternative for analyzing postures and loads is a simulation tool. In particular, the software utilized in this study, JACK and 3DSSPP, allowed user-supplied input for simulation of postures involved in specific tasks. A summary of the features and costs of the aforementioned software is provided in Table 1.

\begin{tabular}{|c|c|c|c|c|c|c|c|c|c|c|c|}
\hline $\begin{array}{c}\text { Software } \\
\text { Features and } \\
\text { Costs }\end{array}$ & 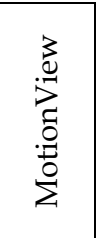 & 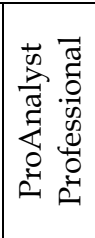 & 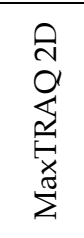 & 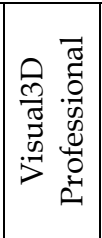 & $\begin{array}{l}0 \\
\frac{1}{a} \\
\substack{x \\
\Sigma}\end{array}$ & $\sum_{\omega}$ & 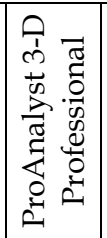 & $\begin{array}{l}\hat{\text { जि }} \\
\text { कि }\end{array}$ & $\begin{array}{l}\text { v } \\
\text {. }\end{array}$ & $\begin{array}{l}\text { च } \\
0 \\
0 \\
3 \\
0 \\
0 \\
0 \\
01\end{array}$ & $\begin{array}{l}\text { ते } \\
0 \\
\infty \\
\text { ¿ }\end{array}$ \\
\hline 2D Analysis & $x$ & $x$ & $x$ & $x$ & $x$ & $x$ & $x$ & $x$ & $x$ & $x$ & $x$ \\
\hline $\begin{array}{c}\text { 3D } \\
\text { Analysis }\end{array}$ & & & & $X$ & $X$ & $X$ & $X$ & $X$ & $X$ & & $X$ \\
\hline $\begin{array}{l}\text { Camera } \\
\text { Required }\end{array}$ & $X$ & $X$ & $X$ & & $x$ & $X$ & $x$ & & OPT & & OPT \\
\hline $\begin{array}{c}\text { Allows Import } \\
\text { of video files }\end{array}$ & & & $X$ & & $X$ & & & & & & \\
\hline $\begin{array}{l}\text { Multiple } \\
\text { Cameras }\end{array}$ & & & & $X$ & $x$ & $x$ & $X$ & & OPT & & OPT \\
\hline \begin{tabular}{|c|} 
High Speed or \\
High Resolution \\
Cameras
\end{tabular} & & $X$ & & $X$ & & $X$ & $X$ & & OPT & & OPT \\
\hline $\begin{array}{l}\text { Calibration } \\
\text { Equipment }\end{array}$ & & & & $X$ & $X$ & $X$ & $X$ & & OPT & & OPT \\
\hline Limited to & $X$ & & & & & & & & & & \\
\hline \begin{tabular}{|c|} 
Existing MoCap \\
Required
\end{tabular} & & & & $X$ & & $X$ & & & OPT & & OPT \\
\hline Muscle Data & & & & & & $X$ & & & & & $X$ \\
\hline System Cost & $\$ 1180$ & $\$ 9595$ & $\$ 695$ & $\$ 15995$ & $\$ 4995$ & $\mathrm{~N} / \mathrm{A}$ & $\$ 14995$ & $\$ 1495$ & $\$ 2400$ & $\$ 1500$ & $\mathrm{~N} / \mathrm{A}$ \\
\hline
\end{tabular}

Table 1. Summary of Commonly Available Biomechanical Software.

\section{Methodology}

The methodology used in this study can be divided into three parts: Selection of Tasks, Tools Used, and Procedures and Analysis. All three of the projects utilized video or photos of the tasks as the basis for the modeling. Some of these photos were taken by the researchers and some were retrieved from the internet. The primary methods of research included internet searches, references to Ergonomics and Biomechanics texts and course notes, search of library archives for relevant research, and creation of simulations using the software in the Ergonomics Laboratory of the University of Central Florida 
The Ergonomic Study in Waste Collection also utilized observational analysis, laboratory analysis and a review of historical data, where surveys were conducted for solid waste collectors and safety personnel of different waste companies in Orlando, Florida to understand the factors affecting waste collectors' safety.

\subsection{Selection of subjects}

Subjects for this study were selected from a population of university students and practitioners. The subjects were selected as components of the three research projects. The tasks identified for evaluation were necessary elements of task performance for the projects and also provided an opportunity for the comparative analysis. The identified tasks contained "task elements" that were used to create simulations and generate data with regard to loads, balance, strength exertion, posture and other task performance descriptors. The tasks which were simulated are described below.

\subsubsection{Solid waste collection project}

\subsubsection{Task 1: Lifting of a full waste container}

Manual lifting of waste containers expose the waste collectors to severe ergonomic risks, repeating this heavy lifting several times during the day lead to musculoskeletal disorders and injuries. This task was broken down into three poses and will be explained in the analysis section.

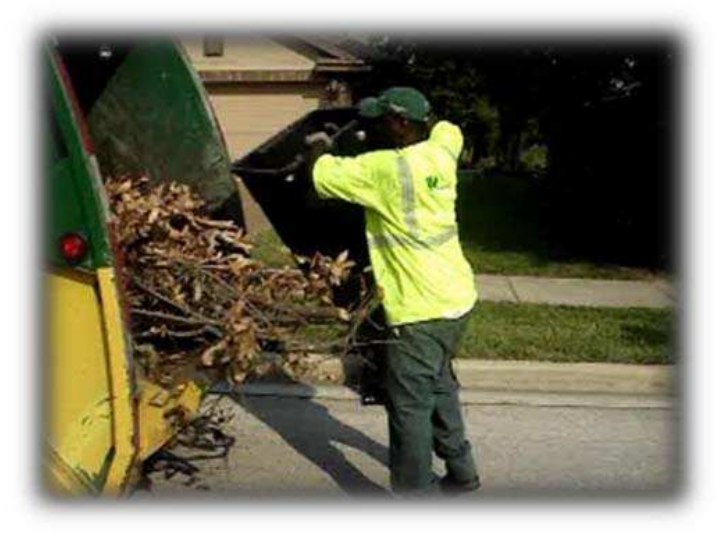

Fig. 2. Lifting the Waste Container Task.

\subsubsection{Task 2: Dumping of a full waste container}

As per the survey that was conducted with the waste collectors, the estimated average container weight is 40 to 60 pounds. Dumping the container that is filled with waste requires awkward postures especially on the lower back region. 


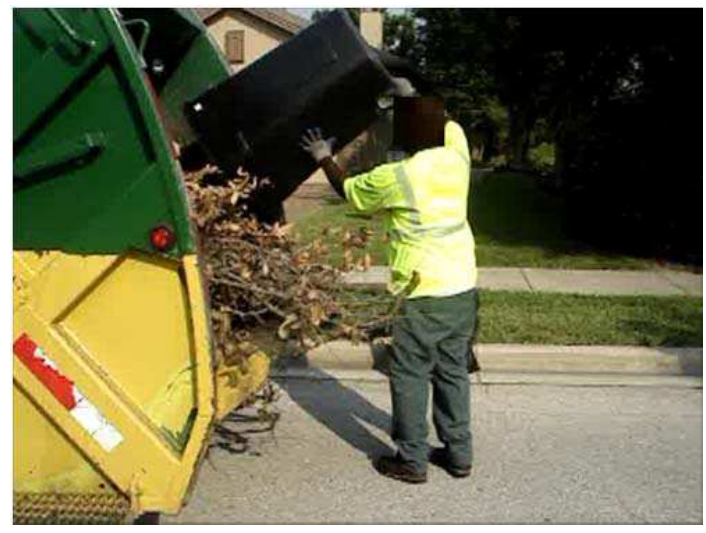

Fig. 3. Dumping the Garbage Container into the Back of the Truck.

\subsubsection{Disaster management project}

\subsubsection{Task 1: Supply distribution}

This task shows the awkward shoulder and arm angles at which supplies are sometimes lifted and moved. This is a typical first responder task.

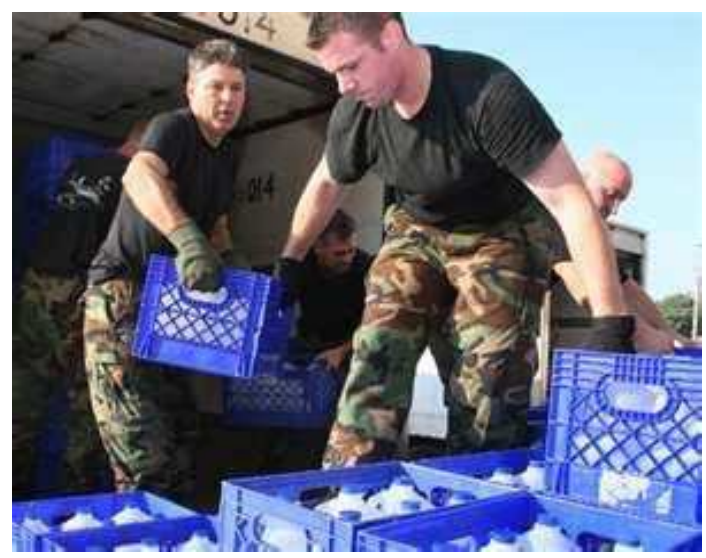

Fig. 4. New Jersey National Guard's Response to Hurricane Katrina, Photo courtesy of pdcbank.state.nj.us

\subsubsection{Task 2: Victim extraction}

Often victim of disasters become trapped in the rubble. Rescues often require awkward and sometimes dangerous postures to keep the victim from incurring additional injury. 


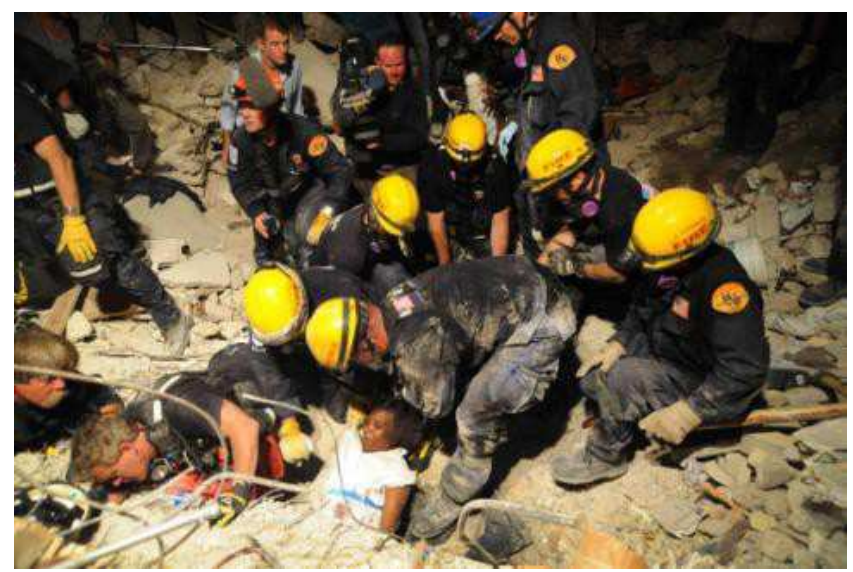

Fig. 5. LA Search and Rescue pull woman out of rubble 12 Jan 2010 Haiti Quake, Photo courtesy of edwardrees.wordpress.com

\subsubsection{Task 3: Moving the injured}

Keeping a victim's head and neck stationary sometimes requires an awkward position by the rescuer. In this case, other rescuers should be taking some of the load at the feet and mid-body so the rescuer does not have to support the entire weight of the victim while keeping the neck stationary.

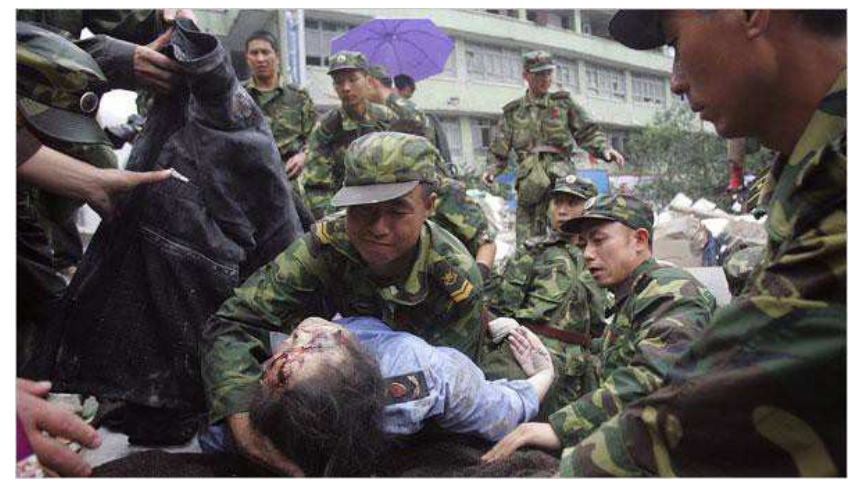

Fig. 6. Rescuers carry injured quake victim from collapsed building, Beichuan County, China. May, 2008, Photo courtesy of nytimes.com

\subsubsection{Interactive gaming project}

For the observational analysis, the experimenter observed and video-recorded the subject performing the Kinect TM Sports "Super Saver" Soccer Game. 

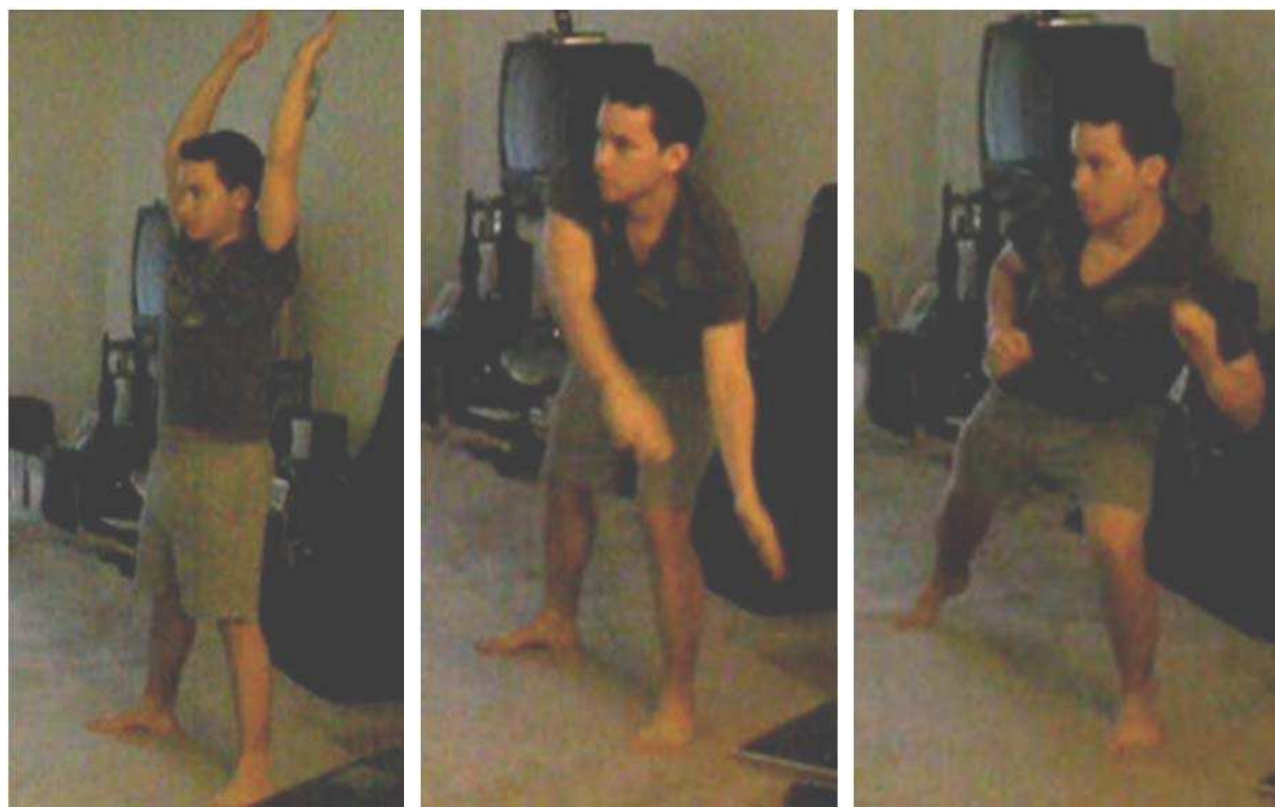

Fig. 7. Still shots of video for Tasks 1-3, respectively.

\subsubsection{Task 1: Overhead catch}

The subject reaches above his head to "catch" the soccer ball and prevent the opponent from scoring.

\subsubsection{Task 2: Low-ball upper-limb save}

The subject reaches across his body to "block" the goal. This movement involves reaching across the midline of the body, resulting in flexion, lateral bending, and rotation of the torso.

\subsubsection{Task 3: Low-ball lower-limb save}

The final pose selected involves the subject's attempt to block the ball with his foot. He extends his right leg, while putting the majority of his weight on the left side of the body.

\subsection{Tools used}

This study primarily required use of two software tools with which to perform biomechanical analysis. Learning the basic functionality of the JACK software required a steep learning curve. Even with extensive man-hours using the manual and the tutorials, the researchers recognize that the software was not utilized to its' maximal functionality. Vendor instructional courses would greatly enhance the users' understanding of all of the features. Despite a rudimentary use of the simulation features, usable data was generated. This data was used to analyze risks associated with the tasks included in the study. This data was also compared with the output generated for the same tasks from another simulation tool, 3DSSPP. 
The following is a summary of the equipment utilized and its purpose:

\subsubsection{Goniometer}

In ergonomics, a goniometer is used to measure, in degrees, active or passive range of motion of applicable joints. This is pertinent to workplace design and functional reach. It can also measure progress in return of range of motion after an injury. For this study, the goniometer was used to measure the angles of limbs for the subject when recreating the solid waste collection tasks in the laboratory.

\subsubsection{D SSPP biomechanical software from the University of Michigan}

3DSSPP predicts static strength requirements for tasks. The program allows user input to simulate the subject postures and loads, and use custom anthropometrics or draw from the installed tables. Output from the software includes spinal compression forces, the percentiles of humans who could perform the task, and data comparisons to NIOSH guidelines, which generate color-coded warnings. The analysis is augmented by graphic illustrations of the positions being studied (University of Michigan, 2010).

The primary feature of interest in the 3DSSPP software, for the purposes of this study, were the low back compression forces, particularly on L5/S1, the region of the spine most prone to lower back injury. These results are displayed in graphical the Summary Analysis Reports where the mark indicates if the force is acceptable (green), caution (yellow) or hazardous (red). The balance reports, moments, and strength analysis reports were also utilized.

\subsubsection{Siemens PLM JACK and the task simulation builder}

"JACK is a human modeling and simulation tool" (JACK) which allows user input simulate a task or environment. "Manufacturing companies in a variety of industries are addressing the human element as a key component of the design, assembly and maintenance of products". JACK utilizes a Task Simulation Builder to enable use of pre-programmed commands to instruct a human model in a virtual 3D environment. This software has a large learning curve, but once the scene is created, the computer will predict the worker movement, utilizing a library of common human movements. The human posturing features clearly incorporate research on prediction of human postures based on any change to the virtual human's posture with regard to variables including hand force exertions, foot positions, center of gravity, head position, and obstacles.

\subsection{Procedure and analysis}

Research including interviews, observations, and literature searches related to each of the three projects yielded preliminary elements to be considered.

For the solid waste collection project, the data collection involved interviewing employees and videotaping of a variety of tasks. The video data was uploaded into a desktop computer and viewed through Windows Movie Maker software. This program allows user to preview the video and under the Tools Tab "Take a Picture from the Preview." The video was viewed at normal speed. The user was then able to go through the video, frame by frame, 
and capture the exact moment to be evaluated and save it as a still photo. This allowed the user to create virtual humans and duplicate the postures and loads of the tasks.

For the disaster management project, still photos of rescue workers were downloaded from a variety of international disasters and used for analysis. The researcher was able to use the photos to create virtual humans and duplicate postures and estimate loads.

The interactive gaming study consists of an observational analysis. The experimenter interviewed the subject to determine his experience with the Xbox Kinect and other video games. Afterwards the subject's anthropometric data was collected. His height was measured with a measuring tape and his weight was measured on a digital scale. For the observational analysis, the experimenter observed and video-recorded the subject performing the Kinect TM Sports "Super Saver" Soccer Game. Next, the video was imported into Windows Live Movie Maker to obtain freeze frames of awkward postures. Next, these snapshots were imported into Adobe Photoshop, where joint angles were determined with the measuring tool.

Once the tasks were identified and the poses selected for simulation, JACK software was used to create a virtual environment to recreate the task. The anthropometric data differed depending upon which of the virtual human models were selected. Hand loads were measured for the waste collection project and those actual loads were used for the objects in the virtual environments. For the disaster management project, the loads were estimated based on user experience. The subject in the interactive gaming project did not have a y hand load. For the purposes of this research, the human posturing techniques were the most useful. This feature allowed users to quickly posture the human model while making predictions of the next movements, based on research of actual human movements and mechanics. The postures from the photos were recreated. An example of the closely simulated posture is shown in Figures 8 through 10.

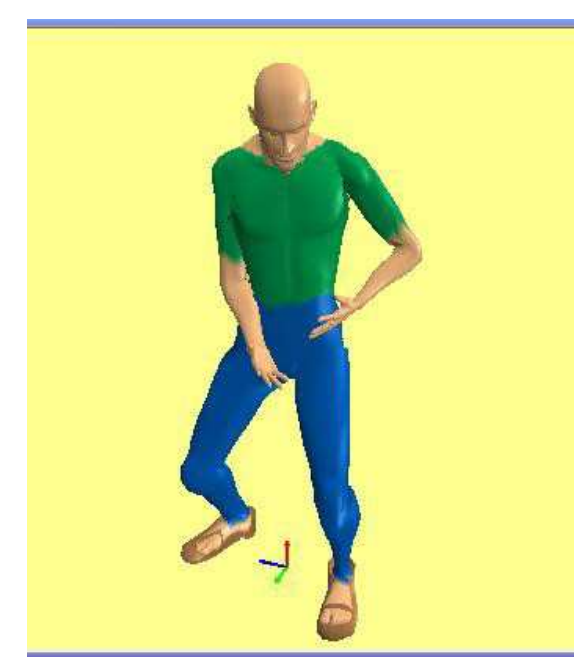

Fig. 8. 3DSSPP Simulation. 


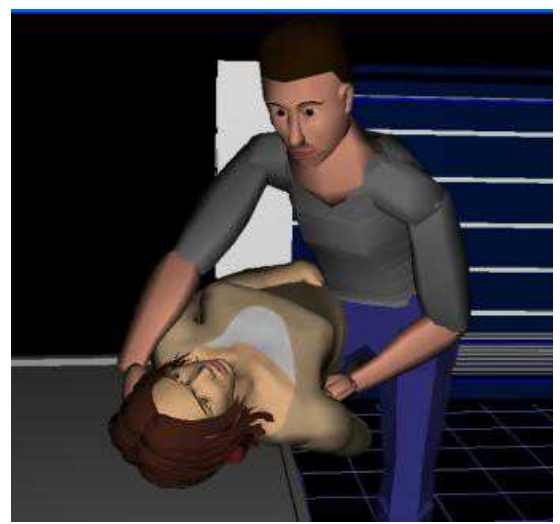

Fig. 9. JACK Simulation of Moving the injured.

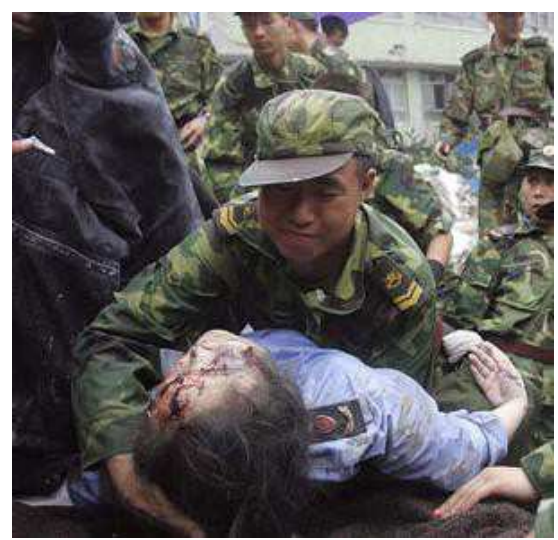

Fig. 10. Moving the Injured.

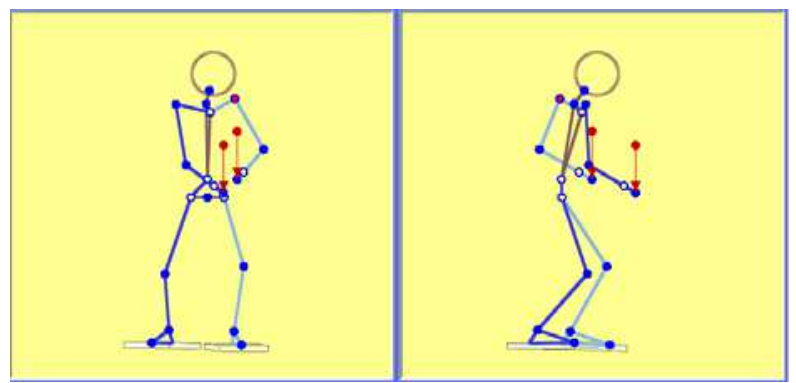

Fig. 11. 3DSSPP Simulation of Moving the Injured.

In the JACK Task Simulation Builder, once the virtual human was manipulated into the correct position, the pose was saved and used in the simulation to be sure the model retained the same pose. The software can help predict the movements either just before or after the pose or poses which are simulated. The reports of interest for this study that were 
generated from JACK included the joint report, the forces report, and the strength analysis report. An attempt was made to utilize the joint angle report to use the JACK values as the starting values to be used in the 3DSSPP simulation. Unfortunately, the joint angles generated by JACK are not the same angles that 3DSSPP requires for input.

The University of Michigan 3DSSPP Biomechanical software was used to analyze the poses, as well. Since 3DSSPP calculates the angles of input from the horizontal, some of the limb orientations and postures of the virtual figure had to be manually manipulated to attain a similar pose. The weights of the objects were the same as those entered in the JACK software. Hand postures were closely matched, as well. An example of the Moving the Injured task simulation in 3DSSPP is seen in Figure 11. The anthropometrics, height and weight, of the virtual figure in JACK were entered into 3DSSPP to keep the variables between the two software packages the same.

The task analysis reports in 3DSSPP predict the percentage of the population who could perform the tasks and were compared with the same percentages generated in JACK. The forces on L4/L5 were also compared. An attempt was made to compare the moments and joint angles, but the degrees of freedom allowed in manually manipulating the virtual figure in 3DSSPP made those factors inconclusive.

3DSSPP gives the Strength Limits for percent capable (percent of the population with sufficient strength) in a graphical format. The green zone is if over $99 \%$ of the population can perform the task. The yellow zone is for $25 \%$ to $99 \%$ of the population and the red zone is if less than $25 \%$ of the population can perform the tasks. JACK gives a red indicator if less than $99 \%$ of the population can perform the task. The percentages were translated from JACK and color-coded to be consistent with the red, yellow, green coding of 3DSSPP to visually clarify the results depicted in the Comparison of JACK and 3DSSPP Output Section of this paper.

\section{Data analysis and results}

Both 3DSSPP and JACK utilize the National Institute of Occupational Safety and Health (NIOSH) lifting guidelines to determine if loads are acceptable. With regard to evaluating whether a simulated posture falls within 'acceptable' limits, the JACK user manual states, "(JACK) Evaluates jobs in real-time, flagging postures where the requirements of a task exceed NIOSH or user-specified strength capability limits." The 3DSSPP User Manual states the following with regard to NIOSH guidelines, "NIOSH recommended limits for percent capable (percent of the population with sufficient strength) are used in the program by default. These values are documented in the Work Practices Guide for Manual Lifting (NIOSH, 1981)" (3DSSPP Manual, p. 3).

Two metrics were used as the primary tools to compare the software: The forces on L4/L5 and the strength capability of the population. The documentation and user guides of the software describe the science behind the calculation of these figures. An attempt was made to compare the moments on L4/L5. Significant variability resulted in the comparison of all of the metrics. Based on the vendor-supplied literature to the software packages, the researchers theorized that the differences in the moment and other 
calculations may have been due to variations in anthropometric data sources, joint angle input and exact posture replication. These topics are discussed in greater detail in the conclusion.

\subsection{Static Strength Prediction percentage capable}

The following quote was taken directly from the JACK user manual: "The Static Strength Prediction (SSP) tool is based on strength studies performed over the past 25 years at the University of Michigan Center for Ergonomics and augmented with data from 250 strengthrelated papers. A collection of strength studies is described in Occupational Biomechanics, 2nd Edition, Chaffin and Anderson, 1991...SSPP was updated for JACK v7.0 to include Wrist Strength using strength equations developed at the University of Michigan Center for Ergonomics. These equations are the same as used in the University's 3DSSPP program and were developed from an analysis of wrist and hand strength data reported in the academic literature (JACK TRAINING MANUAL, p. 18)."

While JACK bases its static strength prediction percentages from data collected at the University of Michigan, 3DSSPP was actually developed by the University of Michigan and utilizes the same population data to calculate the percentages capable for strength. The 3DSSPP Static Strength Prediction Program Version 6.0.3 User Manual (2010) states that, "Population mean strengths...are computed from empirical mean strength equations. The evaluations are based on experimental strength studies by Stobbe (1980); Shanned (1972); Burgraaff (1972); Clarke (1966); Smith and Mayer (1985); Mayer et al (1985); Kishino et al. (1985); Kumr, Chaffin, and Redfern (1985); and many others (3DSSPP Manual, p.84)."

\subsection{Low Back Analysis (forces and moments on L4/L5)}

The JACK User Manual discusses the Low Back Compression Analysis Tool, "the module (that) computes the spinal forces at L4/L5 utilizing the distributed moment histogram $(\mathrm{DMH})$ technique for torso muscle recruitment. (JACK User Manual Version 7.0)...The Low Back Compression Analysis Tool helps evaluate the spinal forces acting on a virtual human's back. The tool tells you compression and shear forces at the L4/L5 vertebral disc, and how the compression forces compare to NIOSH recommended and permissible force limits. The results of a low back Compression analysis can be used to design or modify manual tasks to minimize the risk of low back injuries and conform to NIOSH guidelines. The tool can also pinpoint the exact moments of a lift when the compression forces on a worker's L4/L5 vertebral disc exceed NIOSH force limits. (JACK Low back Analysis Compression Tool Background C: $\backslash$ Program Files $\backslash$ Siemens $\backslash$ Jack_7.0 $\backslash$ library $\backslash$ help $\backslash T A T \_$ Low_back.htm)

The SSPP Manual states that, "the predicted disc compression force shown in the analysis summary can be compared to the NIOSH BCDL of 3400 Newton (3DSSPP Manual, p. 82)." This study focused on L4/L5 compression and moments. This metric is validated by the 3DSSPP manual which states, "Torso muscle moment arms and muscle orientation data for the L4/L5 level have been studied more extensively than at any other lumbar level." (3DSSPP User Manual, p. 4) 


\subsection{Comparison of JACK and 3DSSPP output}

\subsubsection{Subject 1: Solid waste collection project}

\subsubsection{Subject 1, Task 1, Pose 1: Lifting of a full waste container}

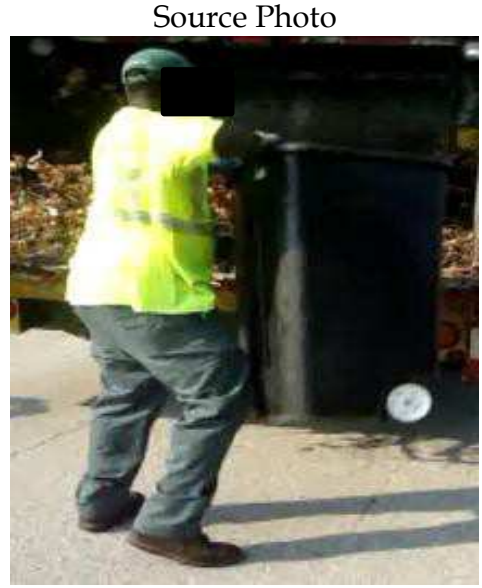

Fig. 12. Lifting Full Waste Container.

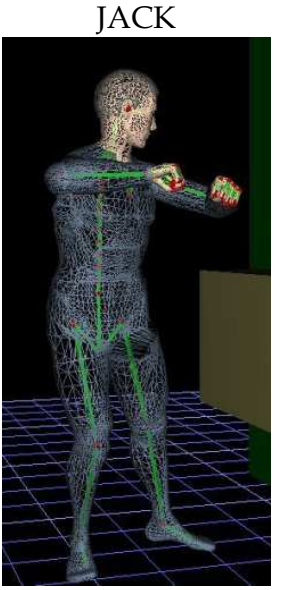

Fig. 13. JACK Simulation - Task 1 , Pose 1.

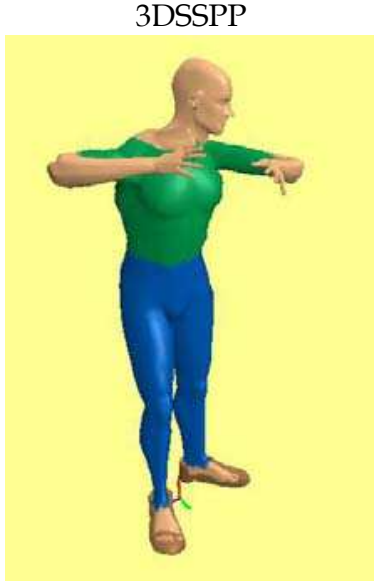

Fig. 14. 3DSSPP Simulation - Task 1, Pose 1.

\begin{tabular}{|l|l|l|} 
& JACK & 3DSSPP \\
\hline L4/L5 Compression Force $(\mathrm{N})$ & 3203 & 2645
\end{tabular}

Table 2. Comparison of JACK and 3DSSPP L4/L5 Forces for Subject 1, Task 1, Pose 1 (See report results in Figures 15 and 18).

\begin{tabular}{|c|c|c|}
\hline & JACK & 3DSSPP \\
\hline Joint & (\% Capable) & (\% Capable) \\
\hline Wrist & 41 & \\
\hline Elbow & 100 & 99 \\
\hline Shoulder & 15 & 92 \\
\hline Torso & 95 & 91 \\
\hline Hip & 97 & \\
\hline Knee & 99 & 86 \\
\hline Ankle & 78 & \\
\hline
\end{tabular}

Table 3. Comparison of JACK and 3DSSPP Strength Capability Summary for Subject 1, Task 1, Pose 1 (See report results in Figures 15 and 17). 


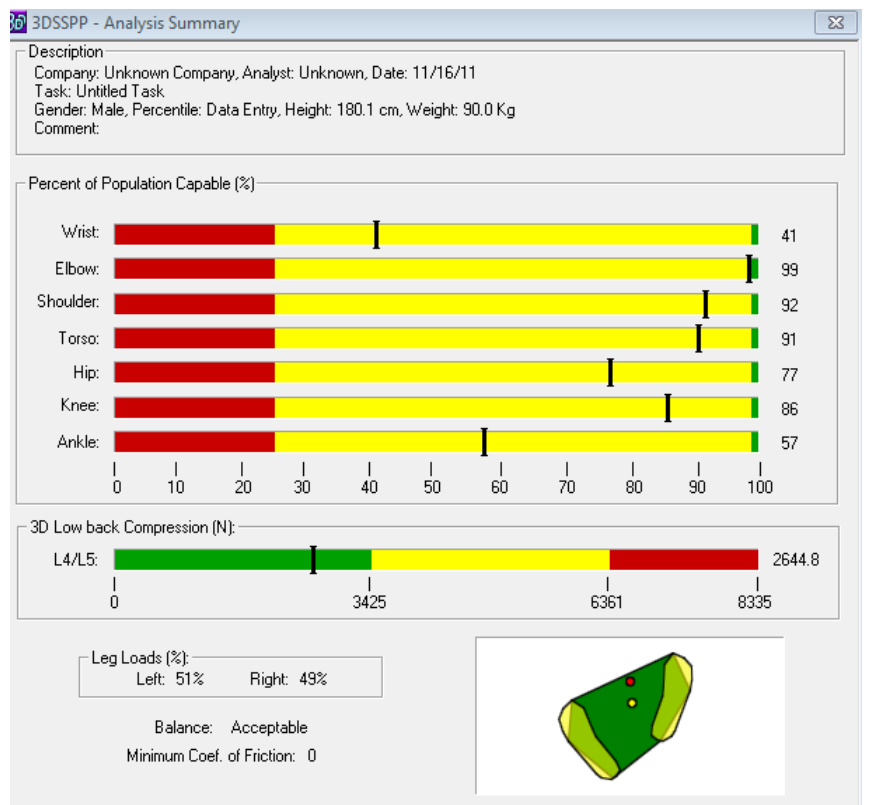

Fig. 15. 3DSSPP Summary Output, Task 1, Pose 1.

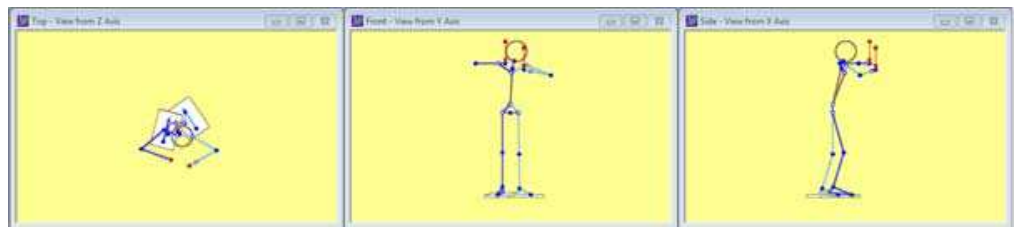

Fig. 16. 3DSSPP Simulation of Limb Angles, Task 1, Pose 1.

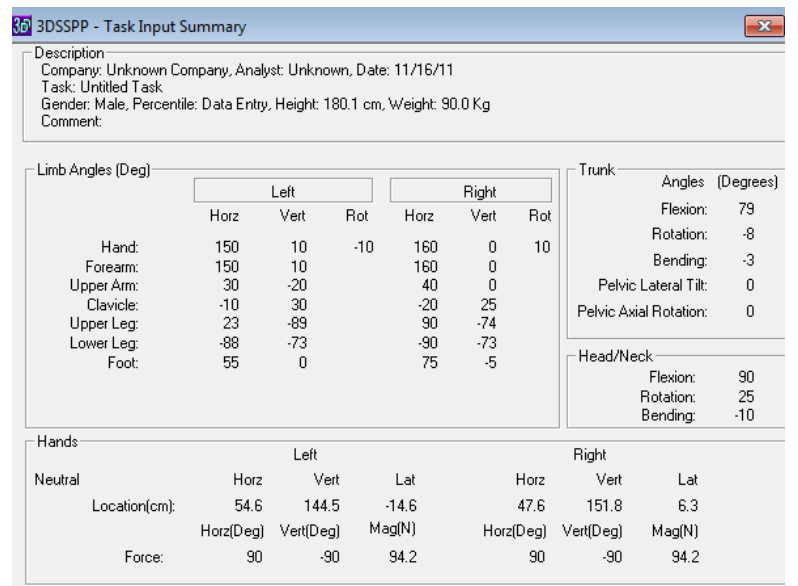

Table 4. 3DSSPP Limb Angle Input, Task 1, Pose 1. 


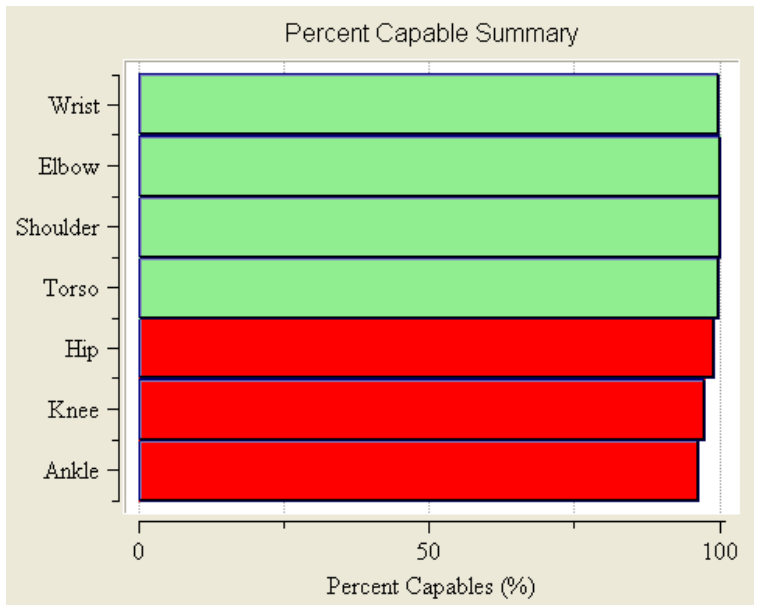

Fig. 17. JACK Output of Percent Capable, Task 1, Pose 1.

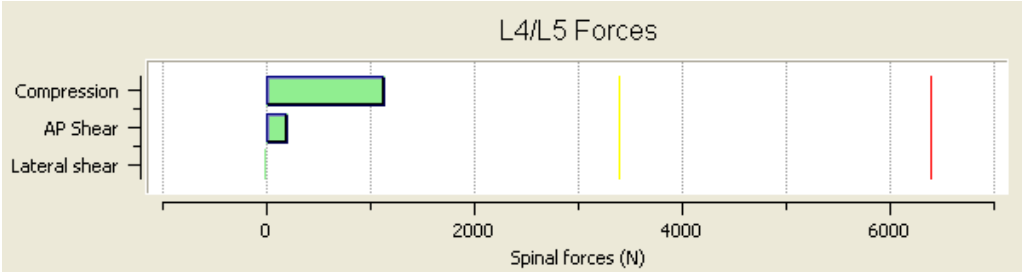

Fig. 18. JACK Output - Forces on L4/L5 for Task 1, Pose 1.

\subsection{Analysis of Subject 1 - Task 1, Pose 1}

Both packages predicted that this pose does not represent severe risk of low back injury since the compression force L4/L5 is below the NIOSH Back Compression Action Limit of $3400 \mathrm{~N}$. In 3DSSPP, the force was $2645 \mathrm{~N}$, while in JACK it was higher by $21 \%$. The waste collector was not bending his torso, so it didn't require high flexion to lift the waste container; the weight of the waste container that was used in the simulation was around 19 $\mathrm{kg}$. The compression force will be higher if the waste collector lifted a heavier container and bent his torso. For the percent capable, it was noted that there is a significant difference for the shoulder joint population strength between both packages, JACK indicated that only 15 $\%$ of the population will be able to perform this pose. On the other hand, 3DSSPP predicted that $92 \%$ of the population is able to perform this pose. This difference may be attributed to the manual manipulation of the postures in 3DSSPP, since this software does not provide the same flexibility to move and twist the shoulder joints as in JACK. Accordingly, for this task, JACK is more applicable to use than 3DSSPP, as it provides more flexibility to manipulate the body joints.

Analysis Recommendations:

The low back compression force of 1123.00 is below the NIOSH Back Compression Action Limit of $3400 \mathrm{~N}$, representing a nominal risk of low back injury for most healthy workers. 


\subsubsection{Subject 1, Task 1, Pose 2: Lifting of a full waste container}
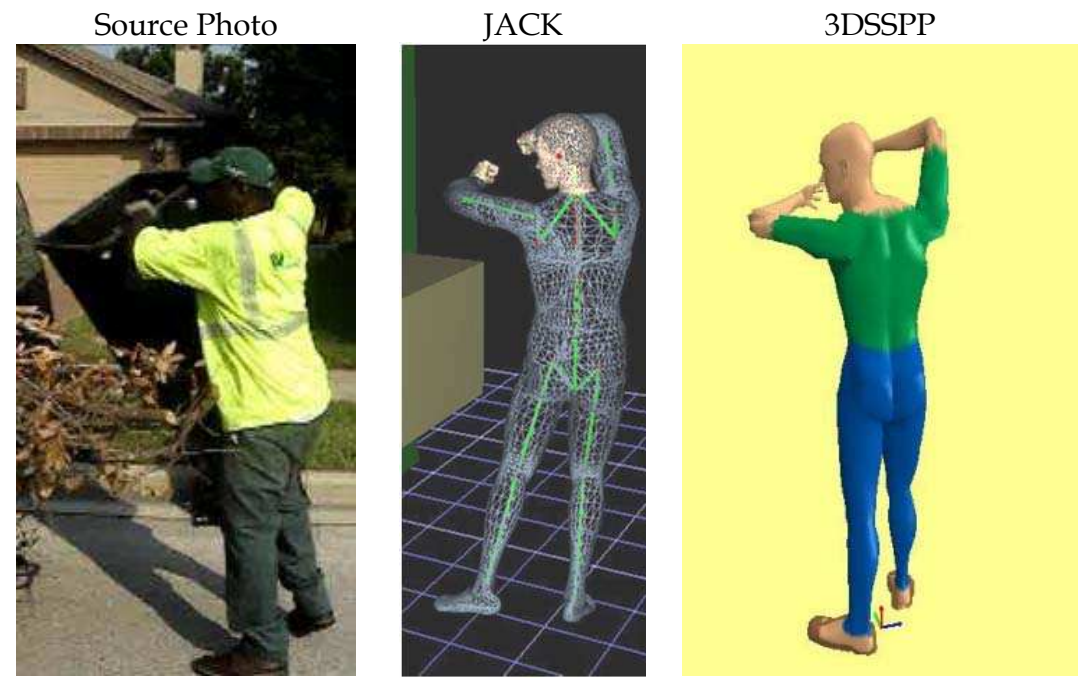

Fig. 19. Lifting Full Waste; JACK; 3DSSPP.

\begin{tabular}{|l|l|l} 
& JACK & 3DSSPP \\
\hline L4/L5 Compression Force $(\mathrm{N})$ & 3243 & 2566
\end{tabular}

Table 5. Comparison of JACK and 3DSSPP L4/L5 Forces for Subject 1, Task 1, Pose 2 (See report results in Figures 20 and 23).

\begin{tabular}{|c|c|c|}
\hline & JACK & 3DSSPP \\
\hline Joint & (\% Capable) & (\% Capable) \\
\hline Wrist & $92 \quad 63$ & \\
\hline Elbow & 44 & 68 \\
\hline Shoulder & 2 & 89 \\
\hline Torso & 99 & 90 \\
\hline Hip & 97 & \\
\hline Knee & 99 & 75 \\
\hline Ankle & $80 \quad 59$ & \\
\hline
\end{tabular}

Table 6. Comparison of JACK and 3DSSPP Strength Capability Summary for Subject 1, Task 1, Pose 2 (See report results in Figures 20 and 22). 


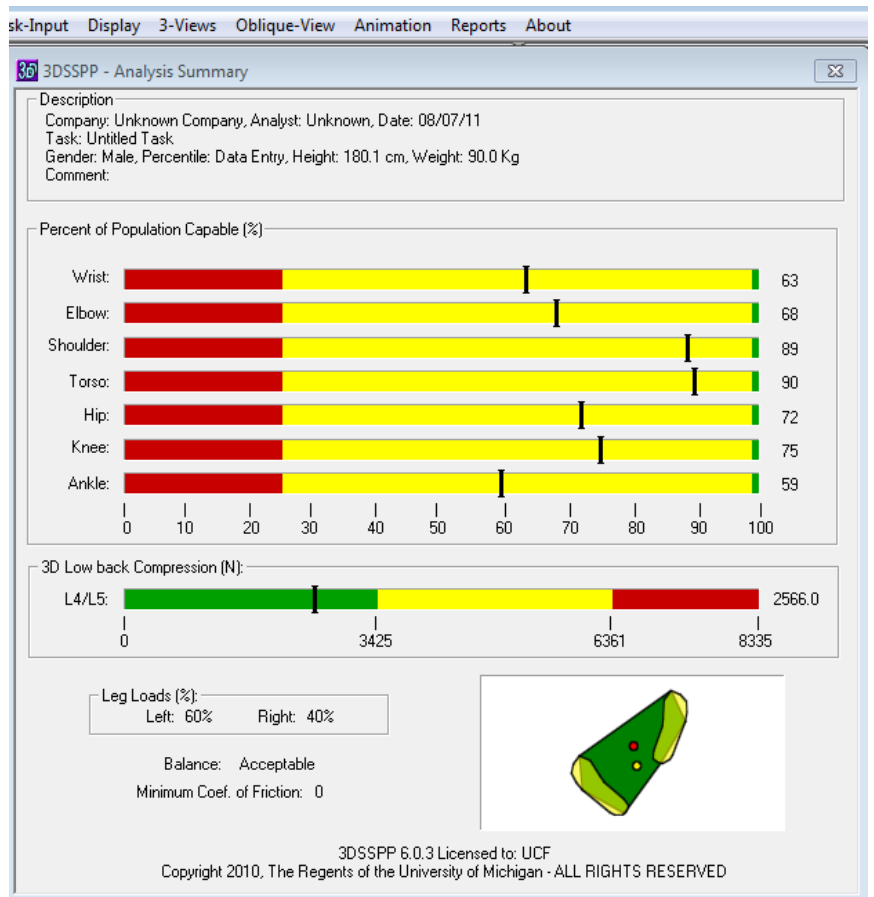

Fig. 20. 3DSSPP Summary Output, Subject 1, Task 1, Pose 2.

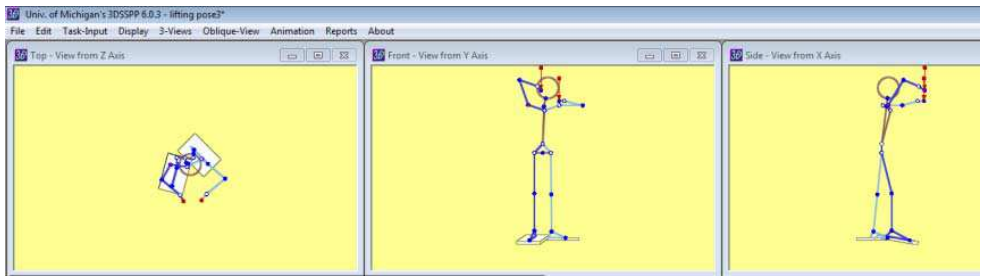

Fig. 21. 3DSSPP Simulation of Limb Angles, Subject 1, Task 1, Pose 2.

\subsection{Analysis of Subject 1 - Task 1, Pose 2}

Although 3DSSPP and JACK indicated that the compression force L4/L5 for this pose acceptable; the force is high and close to the Back Compression Action Limit. In 3DSSPP the force was $2566 \mathrm{~N}$ while in JACK it was higher by $26 \%$. For the percent capable, similar to the previous pose, it was noticed that there is a significant difference between both packages, for the population strength in the shoulder joint; JACK indicated that only $2 \%$ of the population will be able to perform this pose, while 3DSSPP indicated that $89 \%$ of population will perform this pose. According to the observational analysis and the videos, it was noticed that this task requires lifting the garbage container by elevating the shoulder and upper arms at high distance, representing an awkward posture. It was easier to manipulate and rotate the shoulder and the upper arm joints on JACK than 3DSSPP. For the other joints, both packages indicated that they would fall within the yellow zone. 


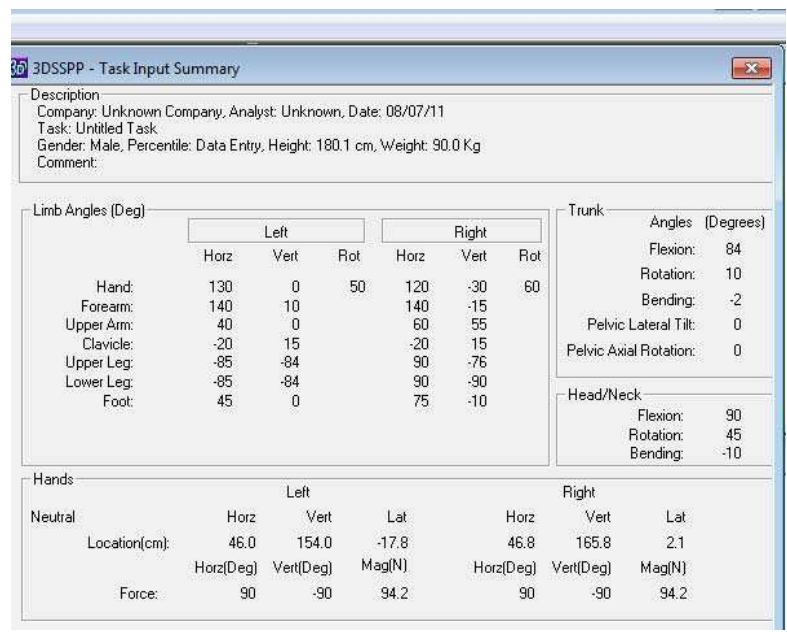

Table 7. 3DSSPP Limb Angle Input, Subject 1, Task 1, Pose 2.

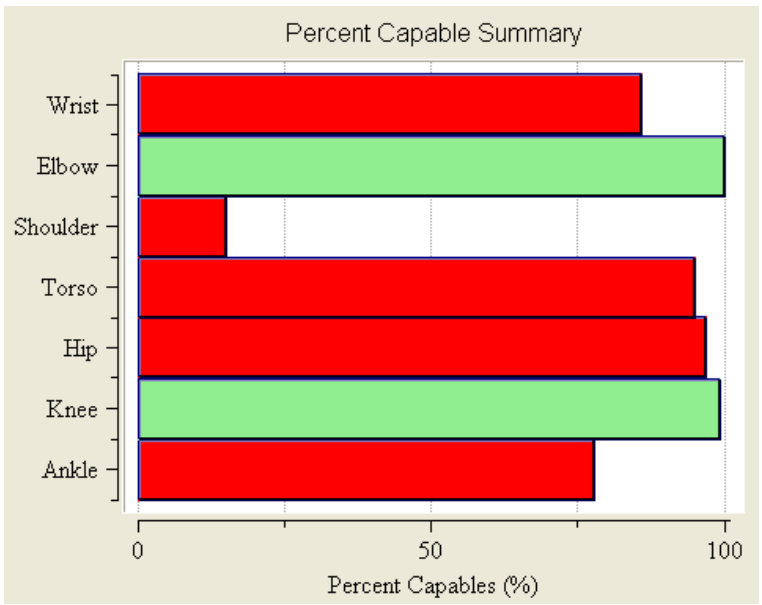

Fig. 22. JACK Output of Percent Capable, Subject 1, Task 1, Pose 2.

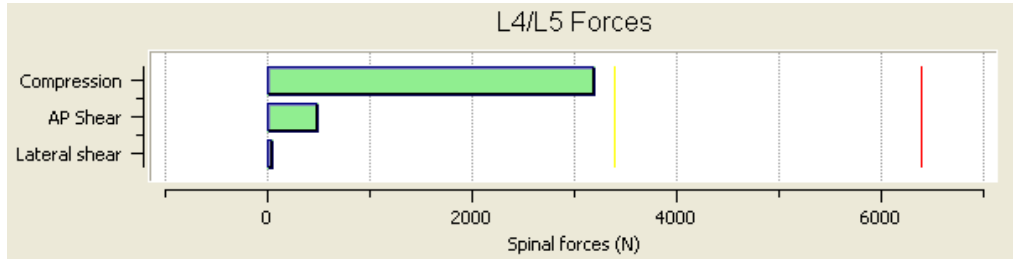

Fig. 23. JACK Output - Forces on L4/L5 for Subject 1, Task 1, Pose 2. 
Analysis Recommendations: The low back compression force of 3203.00 is below the NIOSH Back Compression Action Limit of $3400 \mathrm{~N}$, representing a nominal risk of low back injury for most healthy workers.

\subsubsection{Subject 1, Task 2: Dumping a full waste container}

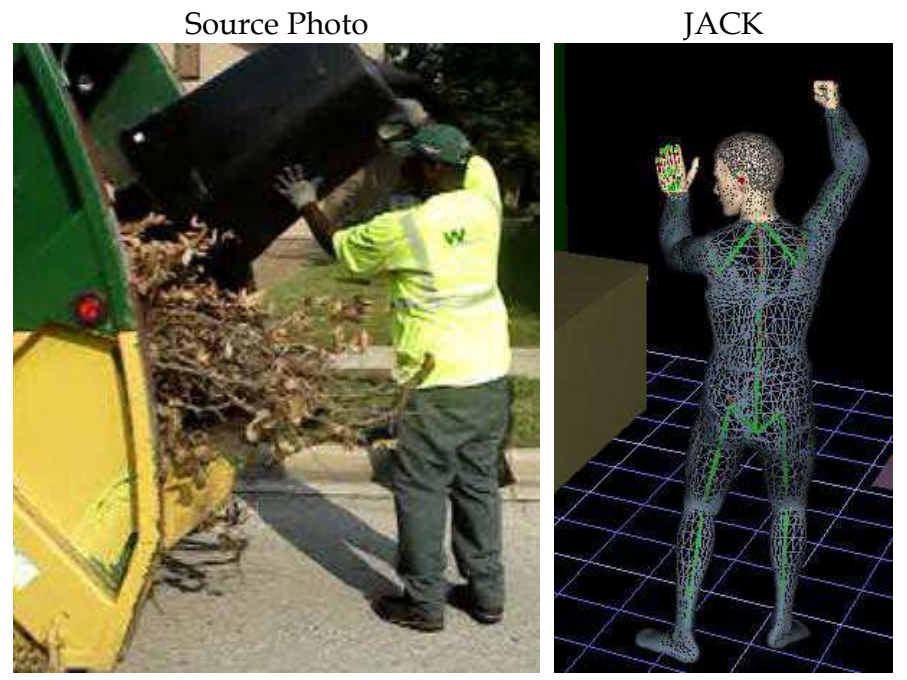

3DSSPP

Fig. 24. Dumping Full Waste; JACK, 3DSSPP.

$\begin{array}{lll} & \text { JACK } & \text { 3DSSPP } \\ \text { L4/L5 Compression Force }(\mathrm{N}) & 3465 & 3491\end{array}$

Table 8. Comparison of JACK and 3DSSPP L4/L5 Forces for Subject 1, Task 2 (See report results in Figures 25 and 28).

\begin{tabular}{|c|c|c|c|}
\hline & \multicolumn{2}{|c|}{ JACK } & 3DSSPP \\
\hline Joint & \multicolumn{2}{|c|}{ (\% Capable) } & (\% Capable) \\
\hline Wrist & 98 & 42 & \\
\hline Elbow & 98 & & 90 \\
\hline Shoulder & 66 & 93 & \\
\hline Torso & 92 & & 11 \\
\hline Hip & 97 & 99 & \\
\hline Knee & 100 & & 70 \\
\hline Ankle & & & \\
\hline
\end{tabular}

Table 9. Comparison of JACK and 3DSSPP Strength Capability Summary for Subject 1, Task 2 (See report results in Figures 25 and 27). 


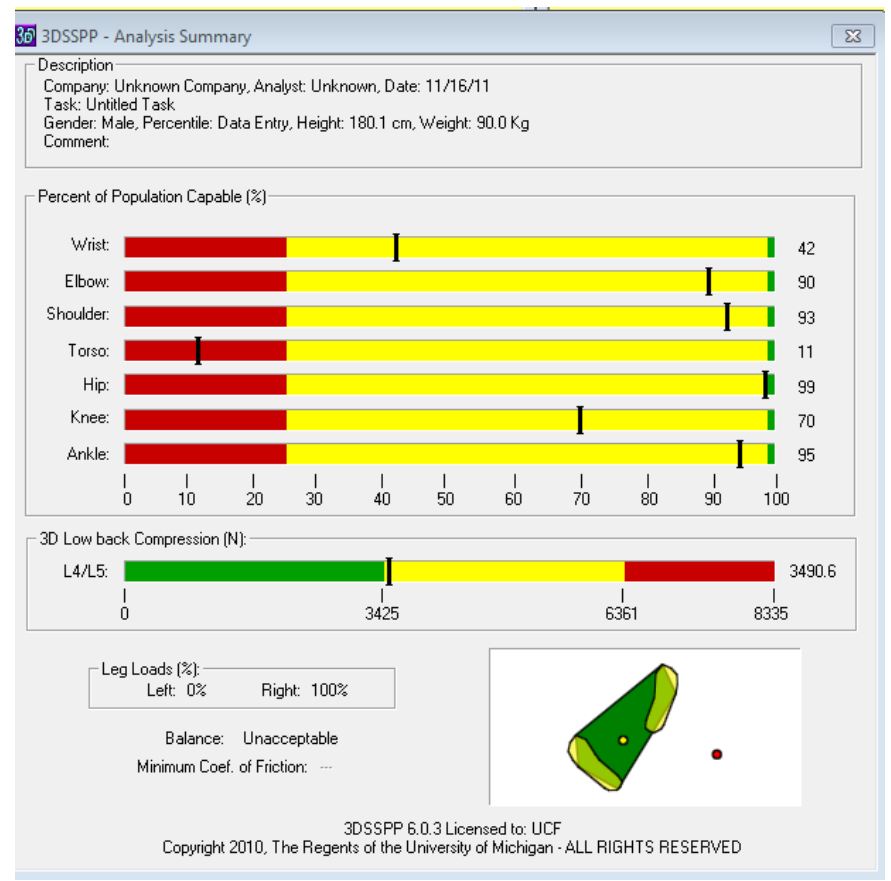

Fig. 25. 3DSSPP Summary Output, Subject 1, Task 2.

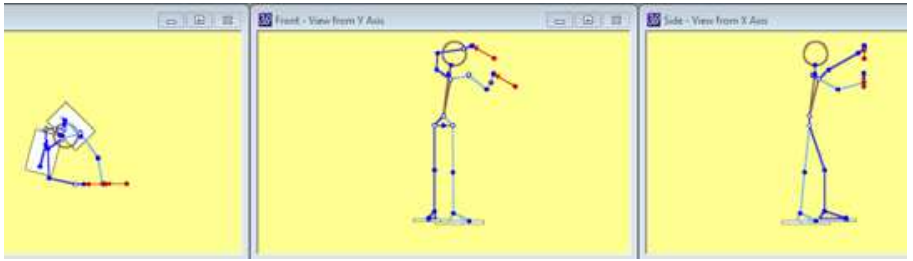

Fig. 26. 3DSSPP Simulation of Limb Angles, Subject 1, Task 2.

\subsection{Analysis of Subject 1 - Task 2}

The results of both simulations concurred that dumping the waste container was the riskiest task for the waste collection workers due not only to the excessive load but also because of the way the worker is lifting the garbage container. The low back compression force in 3DSSPP and JACK exceeds the NIOSH Back Compression Design Limit of 3400N. Workers should avoid twisting while dumping the waste container to avoid awkward postures of the body joints.

The JACK low back analysis report suggests the following ways to reduce the back compressive forces:

1. Reducing the weight of the load.

2. Changing the job environment such that the worker does not need to stoop to lift the load (avoid having to bend over). 
3. Ensuring the load is small, such that it can be held close to the body.

4. Avoiding asymmetric (twisted) postures.

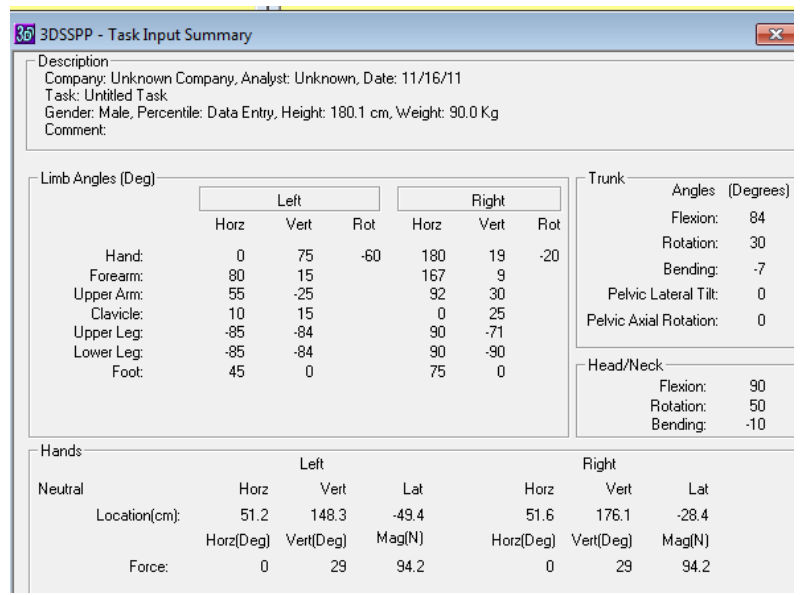

Table 10. 3DSSPP Limb Angle Input, Subject 1, Task 2.

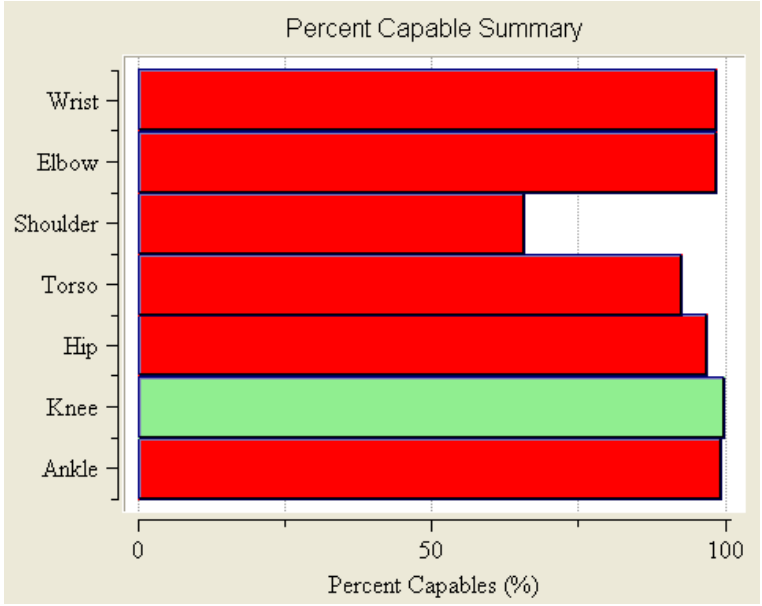

Fig. 27. JACK Output of Percent Capable, Subject 1, Task 2.

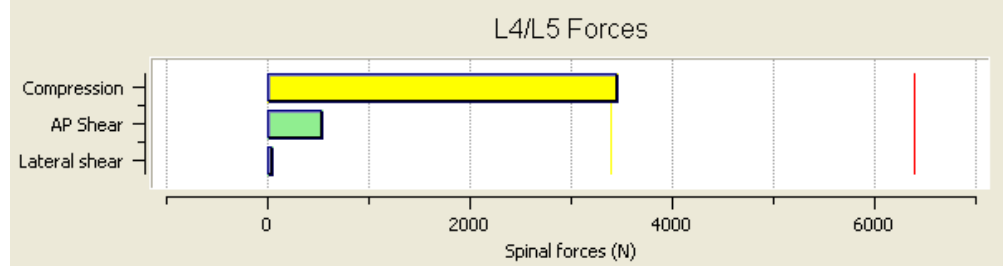

Fig. 28. JACK Output - Forces on L4/L5 for Subject 1, Task 2. 
The percent of the population capable of performing this posture ranges from $11-99 \%$ according to 3DSSPP. The torso area exhibits the most strain; $11 \%$ only of population is capable of performing this task, this percent falls below the NIOSH Upper Limit Value. On the other hand, JACK indicated that $92 \%$ of the population will be able to perform this task with respect to the torso joint. As per the knee joint $100 \%$ of the population will be able to perform this pose, while 3DSSPP indicated that $70 \%$ of population will perform this pose. Also, it was noticed that there is a significant difference between both packages for the wrist joint; JACK indicates that $98 \%$ of population will perform this pose while according to 3DSSPP only $42 \%$ of the population will be able to perform the dumping task. As was mentioned previously, this difference is due the degrees of freedom in manipulating the joints in 3DSSPP. For the other joints, both packages indicated that they would fall within the yellow zone.

\subsubsection{Subject 2: Disaster management project}

\subsubsection{Subject 2, Task 1, Pose 1: Victim extraction}

LA Search and Rescue pull woman out of rubble, 12 Jan 2010 Haiti Quake, Virtual Environment JACK, 3DSSPP.
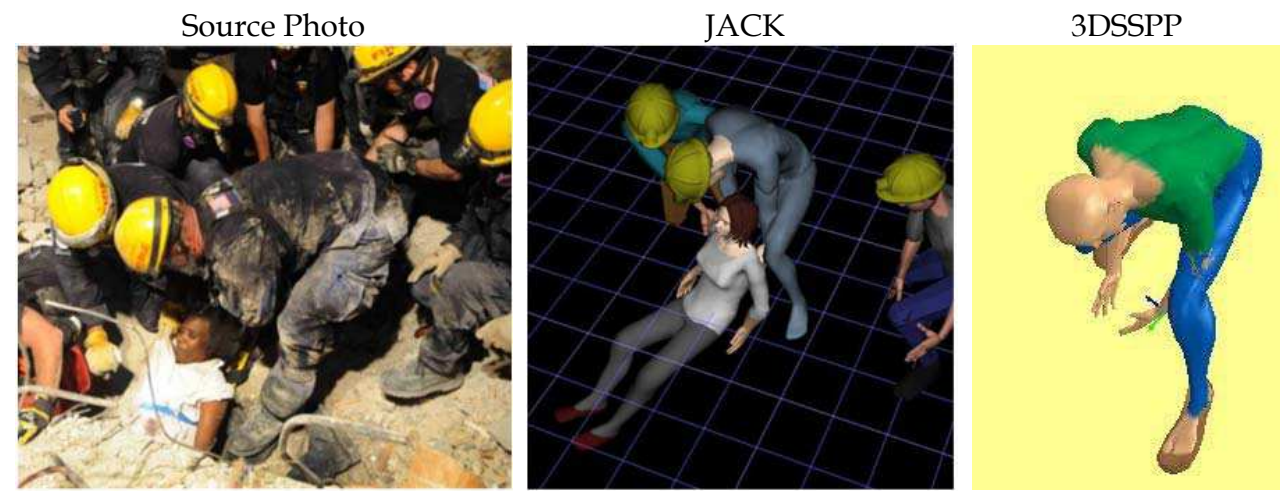

Fig. 29. LA Search and Rescue pull woman out of rubble, 12 Jan 2010 Haiti Quake.

\begin{tabular}{|c|c|c|}
\hline & JACK & 3DSSPP \\
\hline L4/L5 Compression Force (N) & 6658. & 6715. \\
\hline
\end{tabular}

Table 11. Comparison of JACK and 3DSSPP L4/L5 Forces for Subject 2, Task 1 (See report results in Figure 30 and Table 14).

$$
\text { JACK } \quad \text { 3DSSPP }
$$

\begin{tabular}{|c|c|c|}
\hline Joint & (\% Capable) & (\% Capable) \\
\hline Wrist & 13 & \\
\hline Elbow & 74 & 85 \\
\hline
\end{tabular}




\begin{tabular}{l|lcl} 
& JACK & 3DSSPP \\
\hline Shoulder & 53 & 99 & 77 \\
\hline Torso & 80 & & \\
\hline Hip & 96 & 44 & 25 \\
\hline Knee & 86 & & \\
\hline Ankle & 99 & 93 &
\end{tabular}

Table 12. Comparison of JACK and 3DSSPP Strength Capability Summary for Subject 2, Task 1(See report results in Figures 30 and 32).

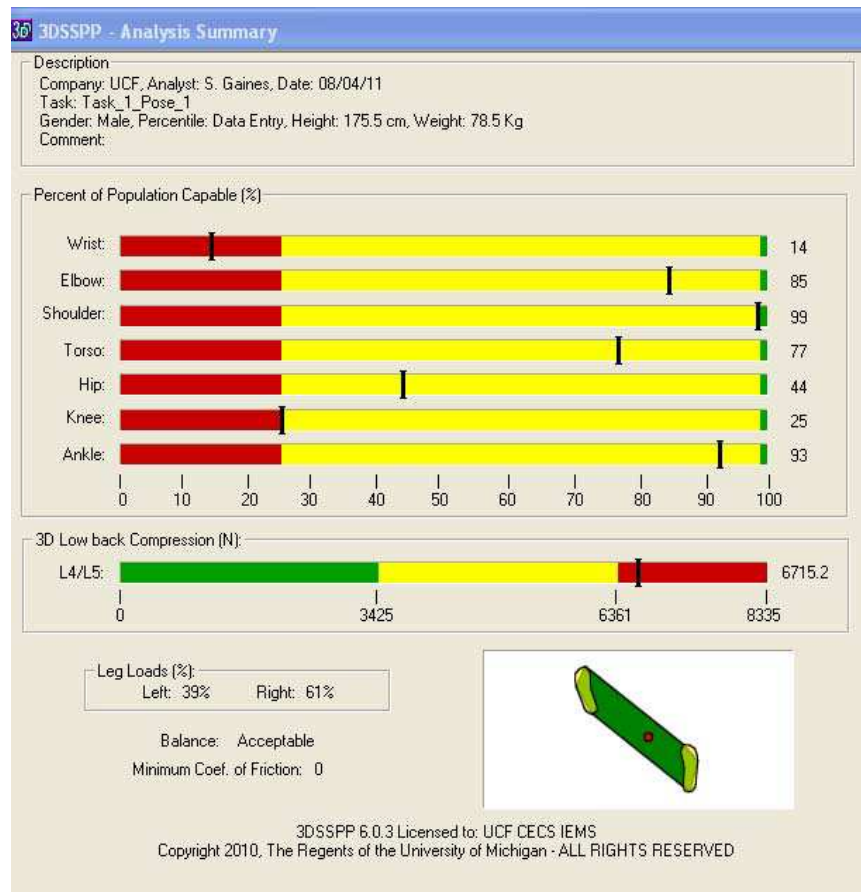

Fig. 30. 3DSSPP Summary Output, Subject 2, Task 1.

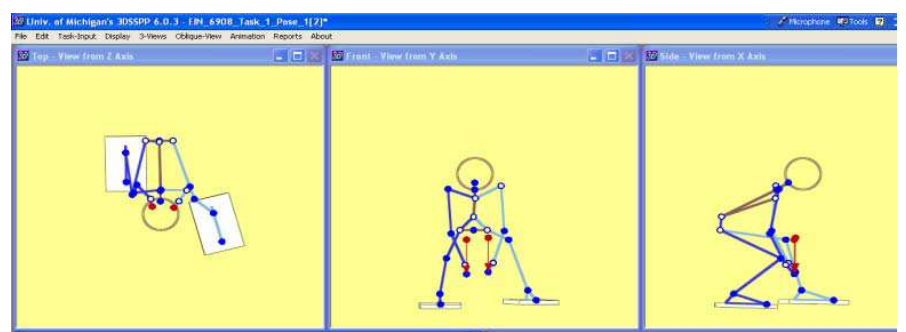

Fig. 31. 3DSSPP Simulation of Limb Angles, Subject 2, Task 1. 


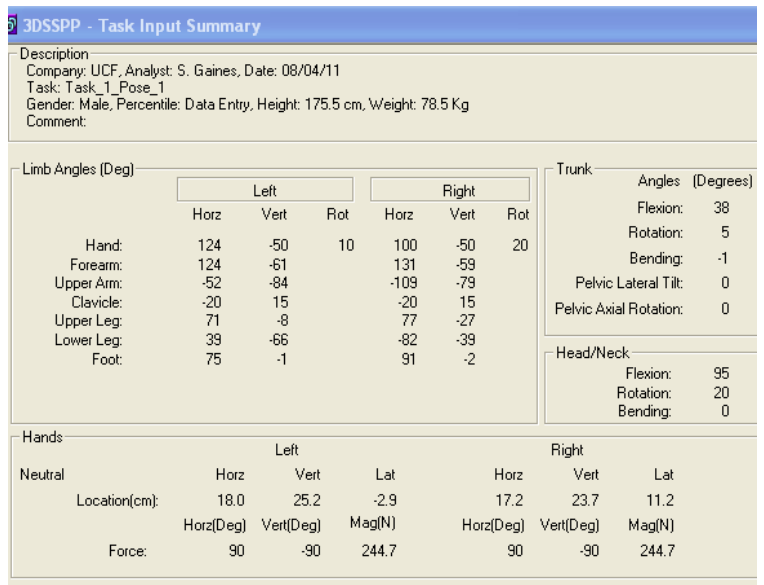

Table 13. 3DSSPP Limb Angle Input, Subject 2, Task 1.

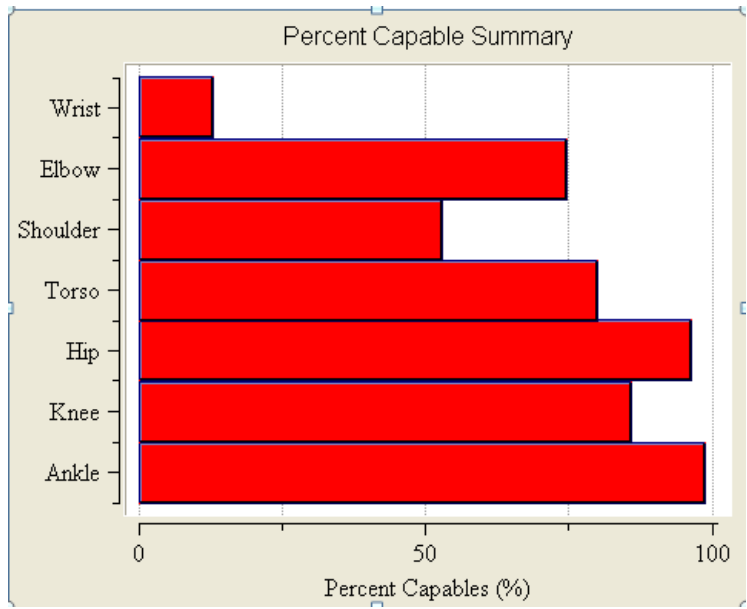

Fig. 32. JACK Output of Percent Capable, Subject 2, Task 1.

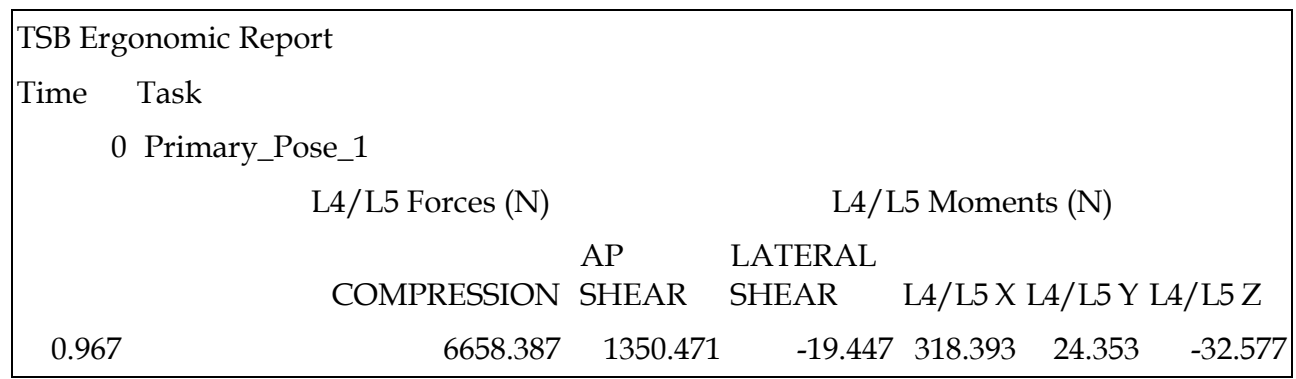

Table 14. JACK TSB Ergonomic Report - Forces on L4/L5, Subject 2, Task 1. 


\subsection{Analysis of Subject 2 - Task 1, Pose 1}

The compression forces on L4/L5 are similar between JACK and 3DSSPP, that is, they are within $57 \mathrm{~N}$ of each other, or less than a $1 \%$ difference. For the percent capable, both packages predicted 7 of the 8 joints would fall within the caution, or yellow zone and one joint would fall within the red zone. These tasks appear to have been simulated in a similar fashion and the output is comparable.

For this task either software package would generate similar results. In both cases, the forces on L4/L5 fall well above the NIOSH recommended upper limit of $3400 \mathrm{~N}$, meaning performance of this task, especially if repeated frequently using these postures and loads will likely result in injury to the first responder. In fact, both of the software packages calculated a force on L4/L5 of greater than the maximum limit allowed by NIOSH of $6400 \mathrm{~N}$. This task should not be performed by only one person. At these angles and loads, at least two people must assist in lifting the load.

Analysis Recommendations: The low back compression force of 3465.00 is above the NIOSH Back Compression Action Limit of $3400 \mathrm{~N}$, representing an increased risk of low back injury for some workers. It is recommended that this job analyzed further for ways to reduce low back forces.

\subsubsection{Subject 2, Task 2, Pose 5: Moving the injured}
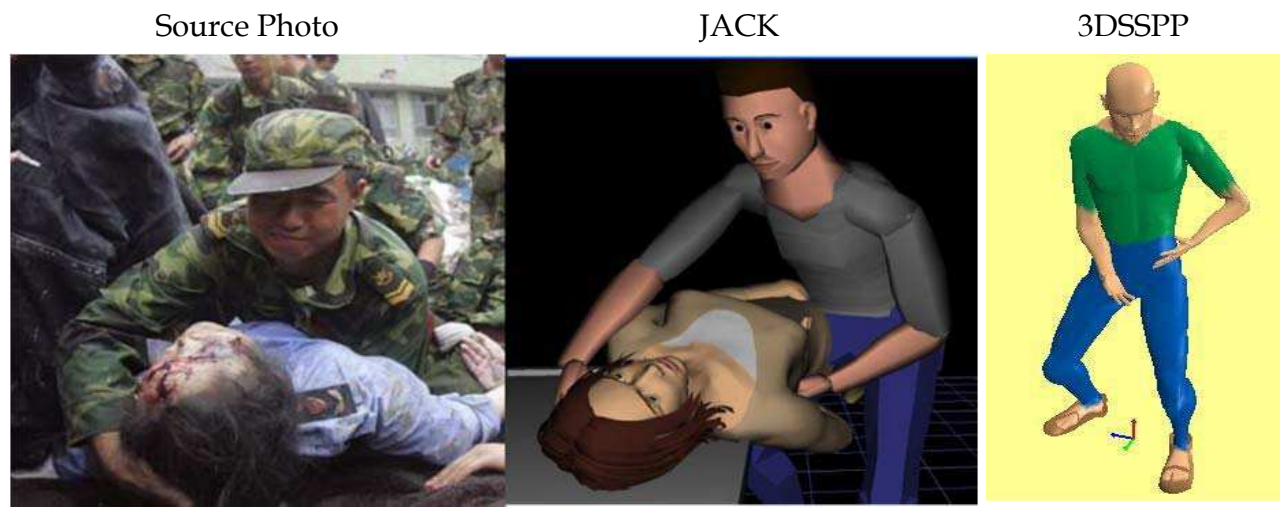

Fig. 33. Rescuers carry injured quake victim from collapsed building in Beichuan County, China. May, 2008.

\begin{tabular}{l|ll} 
& JACK & 3DSSPP \\
L4/L5 Compression Force (N) & 6853. & 6966.
\end{tabular}

Table 15. Comparison of JACK and 3DSSPP L4/L5 Forces for Subject 2, Task 2 (See report results in Figure 34 and Table 18).

\begin{tabular}{l|l|l|} 
Joint & $(\%$ Capable $)$ & $(\%$ Capable $)$ \\
\hline Wrist & 3 & 0
\end{tabular}




\begin{tabular}{ll|l} 
Joint & $(\%$ Capable $)$ & $(\%$ Capable $)$ \\
\hline Elbow & 50 & 0 \\
\hline Shoulder & 0 & 0 \\
\hline Torso & 48 & 20 \\
\hline Hip & 90 & 3 \\
\hline Knee & 99 & 98 \\
\hline Ankle & 87 & 2
\end{tabular}

Table 16. Comparison of JACK and 3DSSPP Strength Capability Summary for Subject 2, Task 2 (See report results in Figures 34 and 36).

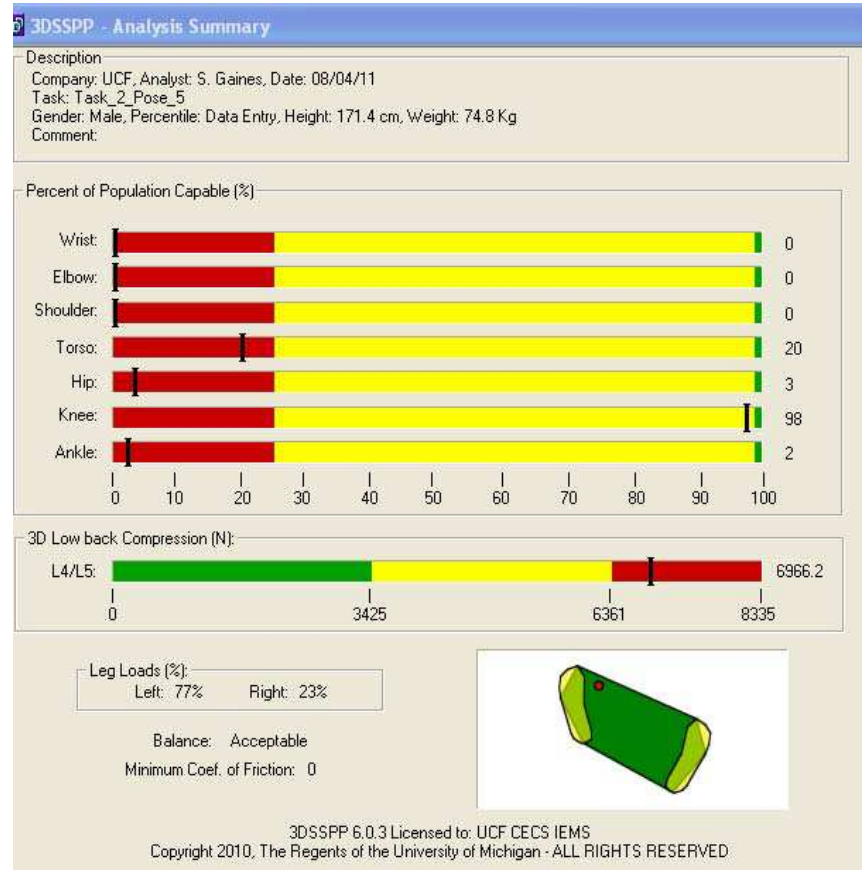

Fig. 34. 3DSSPP Summary Output, Subject 2, Task 2.

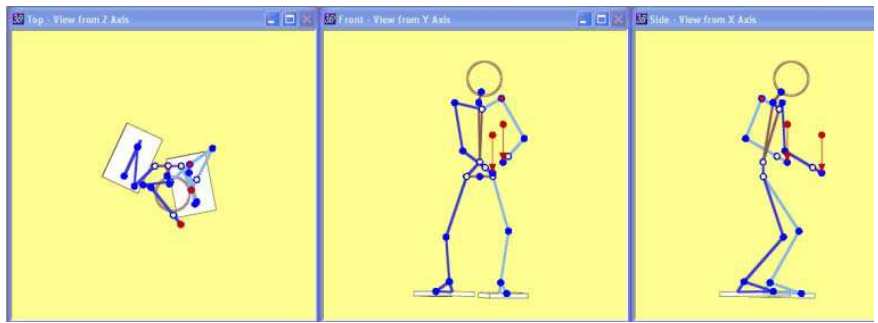

Fig. 35. 3DSSPP Simulation of Limb Angles, Subject 2, Task 2. 


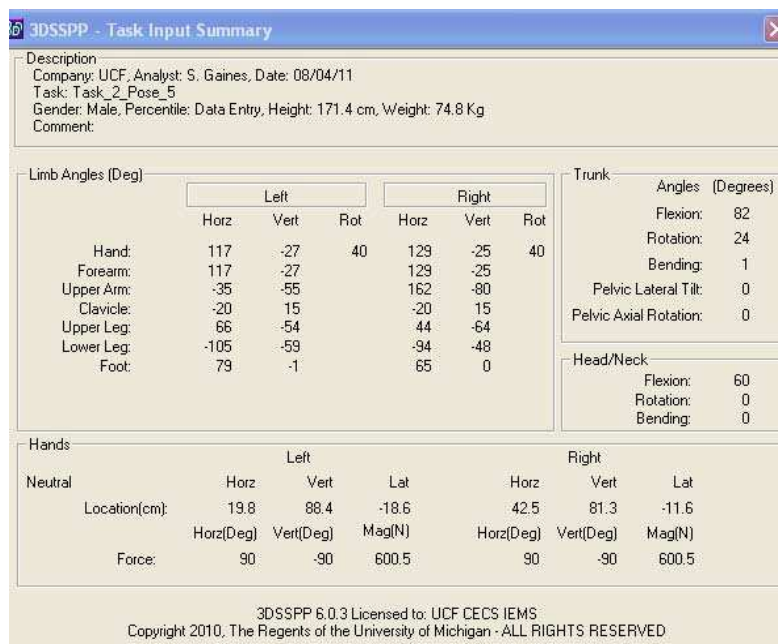

Table 17. 3DSSPP Limb Angle Input, Subject 2, Task 2.

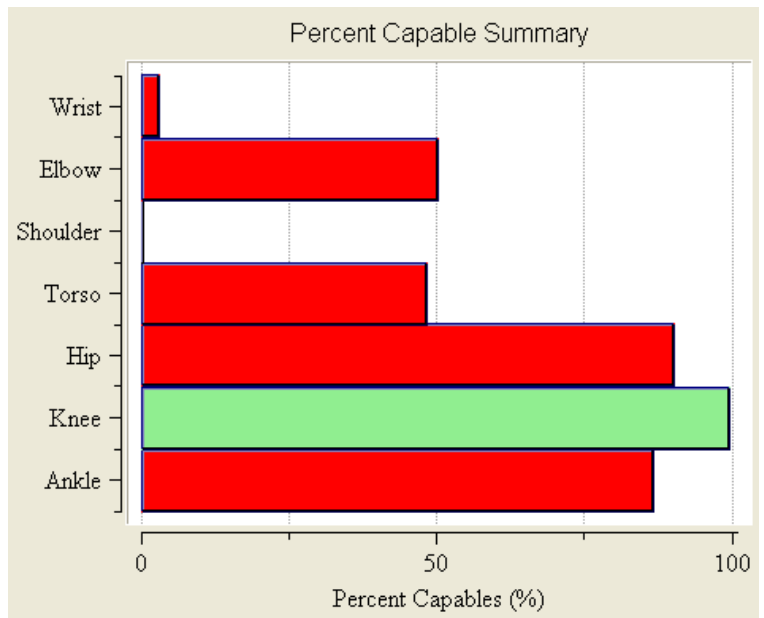

Fig. 36. JACK Output of Percent Capable, Subject 2, Task 2.

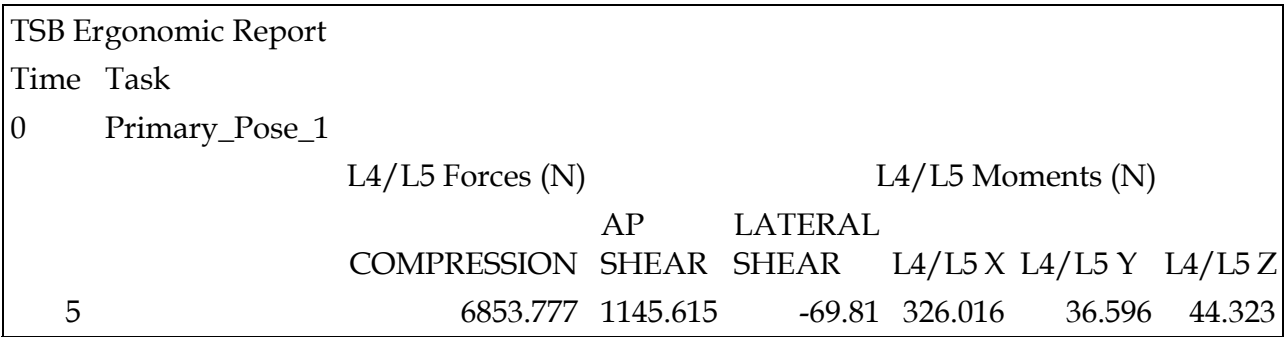

Table 18. JACK TSB Ergonomic Report - Forces on L4/L5, Subject 2, Task 2. 


\subsection{Analysis of Subject 2 - Task 2, Pose 5}

The forces on L4/L5 generated by the two software packages are similar, with results within $2 \%$ of each other. The compression force on L4/L5 calculated by both packages indicates that this task activity is above the maximum allowable NIOSH limit of $6400 \mathrm{~N}$. Essentially this task should not be performed by one person and not in the postures exhibited.

For the percent capable, 3DSSPP predicted this is a more difficult task for the majority of the population to perform than JACK. In fact, 3DSSPP calculated that $5 \%$ or less of the population could perform the task for 5 of the 8 joints analyzed. A review of the overall data found in Figures 34 and 36, indicates that the shoulder, for example, has a $98-100 \%$ capable in all areas except one. That one is noted to be at $0 \%$, so the software automatically accepts the lowest number. The other significant difference in was seen in the hip joint. 3DSSPP said only $3 \%$ of the population could perform the task, while JACK thinks $90 \%$ of the population can perform the task. Manually manipulating the postures in 3DSSPP was the only way to visually achieve the "same" posture. 3DSSPP seems to have less ability to gradually change the postures. When the center of hips is moved for example, all of the other angles changed dramatically.

For this task, JACK appears to be a better simulation tool. It allows more detailed manipulation of hand postures and also gives more flexibility with regard to torso rotations and flexibility. The greater ability to specify the angles of shoulder rotation, elevation, and lift are also pivotal in this task analysis. See Figure 37 for an example of how JACK allows this detailed input. The force on the shoulder was probably one of the greatest, other than on L4/L5 for this task.

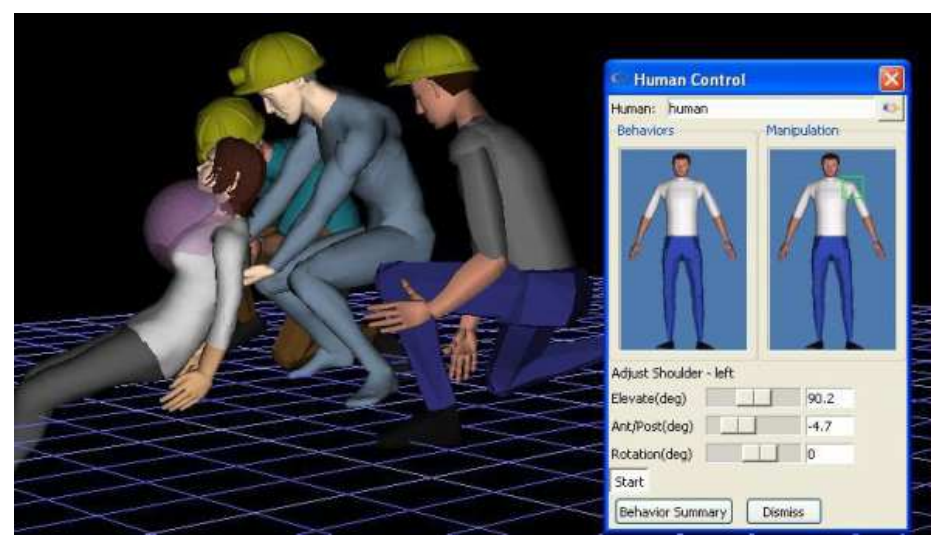

Fig. 37. The Human Control tab in JACK allows greater manipulation of the shoulder joint, pivotal in this task. 


\subsubsection{Subject 2, Task 3, Pose 4: Supply distribution}
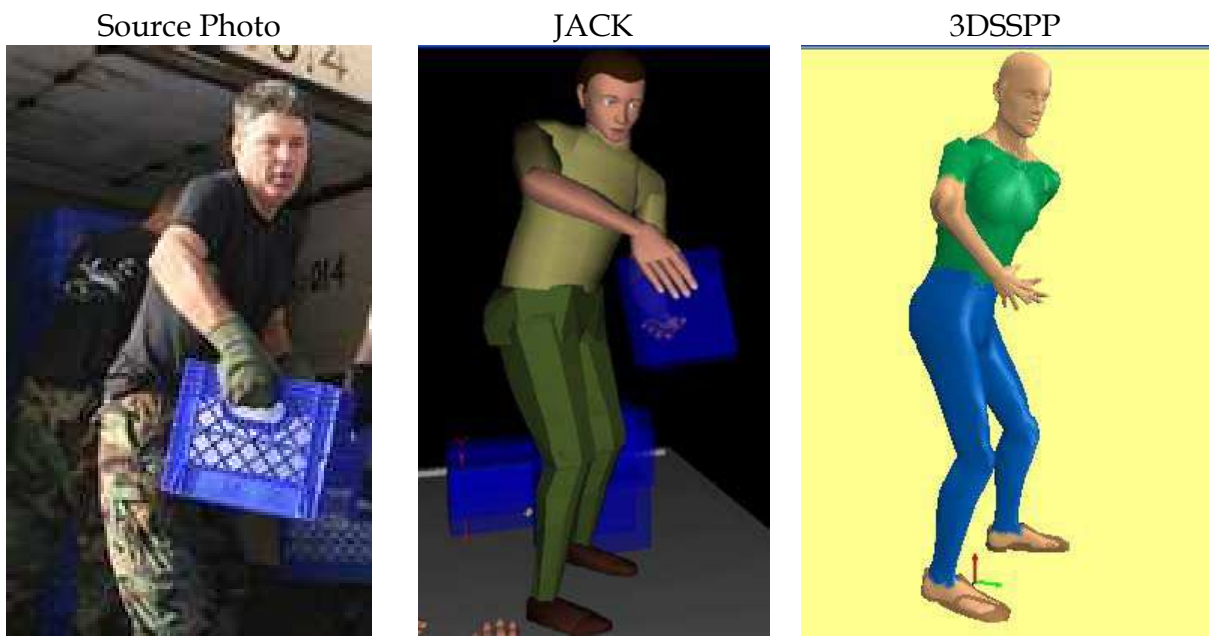

Fig. 38. New Jersey National, Guard's Response to Hurricane Katrina Photo courtesy of pdcbank.state.nj.us

\section{JACK $\quad$ 3DSSPP}

L4/L5 Compression Force (N)

2626. 2461.

Table 19. Comparison of JACK and 3DSSPP L4/L5 Forces for Subject 2, Task 3 (See report results in Figure 39 and Table 22).

\begin{tabular}{llll}
\multicolumn{3}{l}{ JACK } & 3DSSPP \\
Joint & \multicolumn{2}{l}{$\begin{array}{l}\text { (\% Capable) } \\
\text { (\% Capable) }\end{array}$} & \\
\hline Wrist & 99 & 67 & 98 \\
\hline Elbow & 100 & & \\
\hline Shoulder & 99 & 99 & 96 \\
\hline Torso & 98 & & \\
\hline Hip & 98 & 91 & 99 \\
\hline Knee & 100 & &
\end{tabular}

Table 20. Comparison of JACK and 3DSSPP Strength Capability Summary for Subject 2, Task 3. (See report results in Figures 39 and 41). 


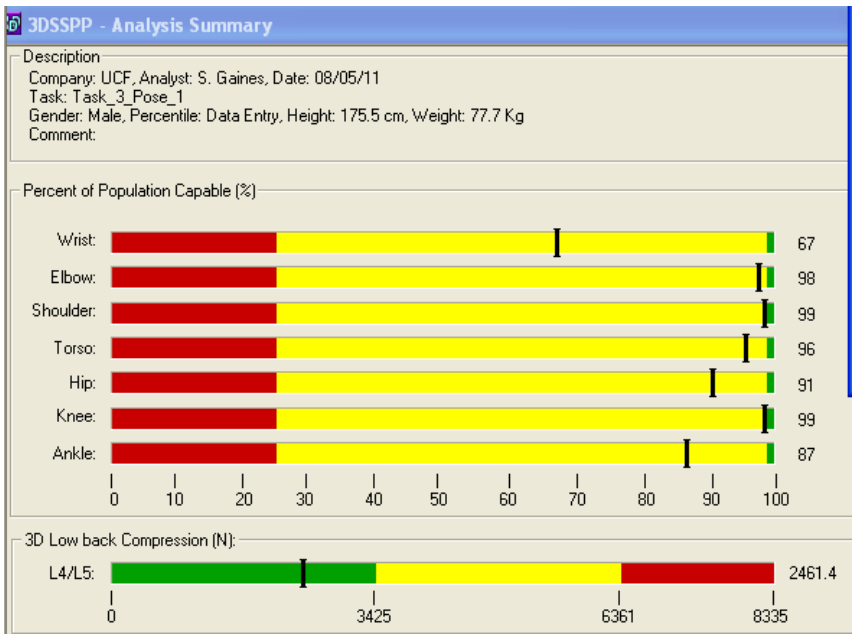

Fig. 39. 3DSSPP Summary Output, Subject 2, Task 3.

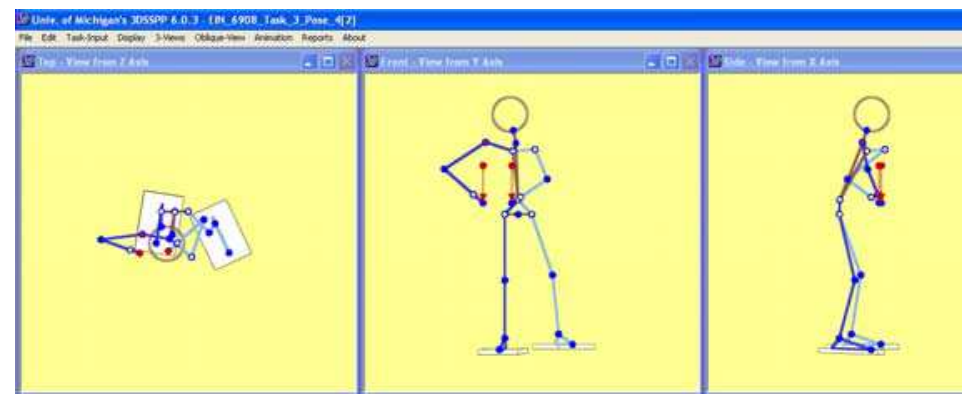

Fig. 40. 3DSSPP Simulation of Limb Angles, Subject 2, Task 3.

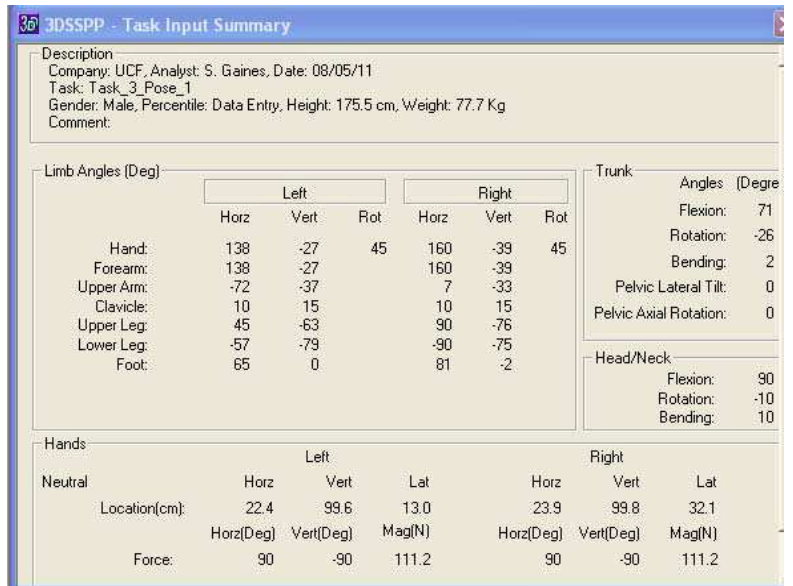

Table 21. 3DSSPP Limb Angle Input, Subject 2, Task 3. 


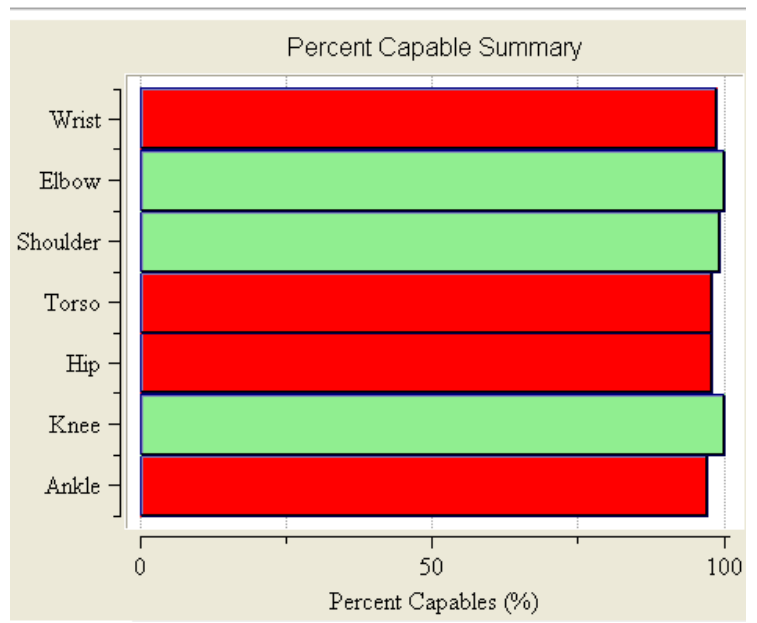

Fig. 41. JACK Output of Percent Capable, Subject 2, Task 3.

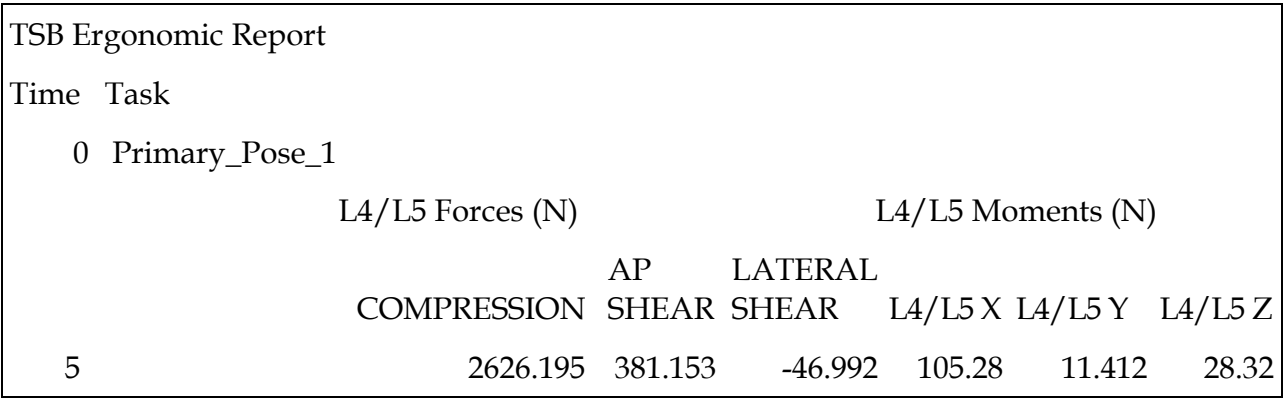

Table 22. JACK TSB Ergonomic Report - Forces on L4/L5, Subject 2, Task 3.

\subsection{Analysis of Subject 2 - Task 3, Pose 4}

Both JACK and 3DSSPP generated similar L4/L5 compression force calculations, and while slightly different, the forces on L4/L5 for this task fell below the NIOSH recommended upper limit of $3400 \mathrm{~N}$ for both packages. The frequency with which this task may be repeated was not considered, and would inevitably generate a fatigue factor if, for example, an entire truckload of supplies at this weight using this posture were unloaded. Both software packages calculated that the percent of the population which could perform this task was over $90 \%$ for every joint except one (3DSSPP said only $67 \%$ of the population could perform these wrist manipulations). Either package would be able to adequately simulate this task. 5.3.3 SUBJECT 3: INTERACTIVE GAMING PROJECT 


\subsubsection{Subject 3, Task 1: Overhead Catch}
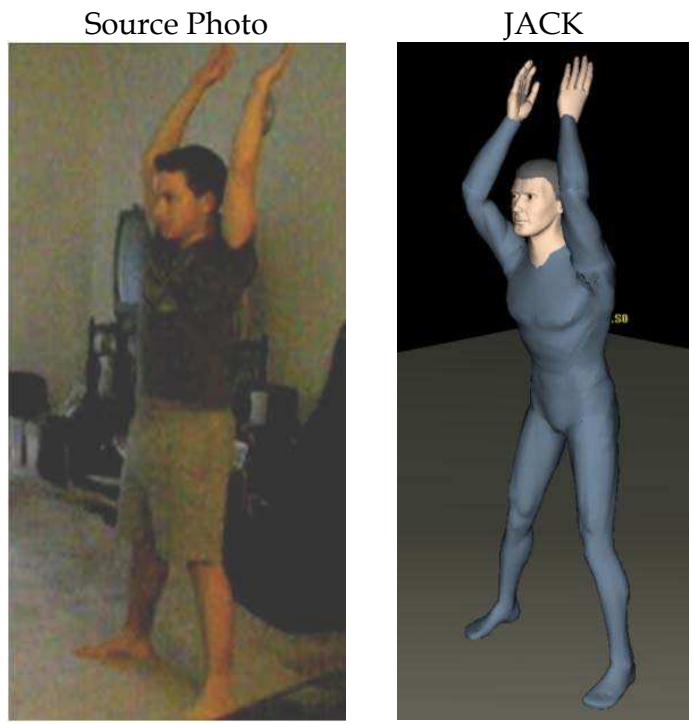

\section{DSSPP}

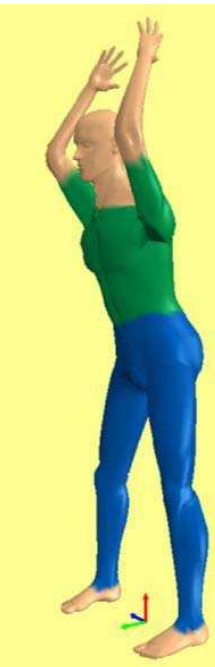

Fig. 42. Overhead Catch; JACK; 3DSSPP.

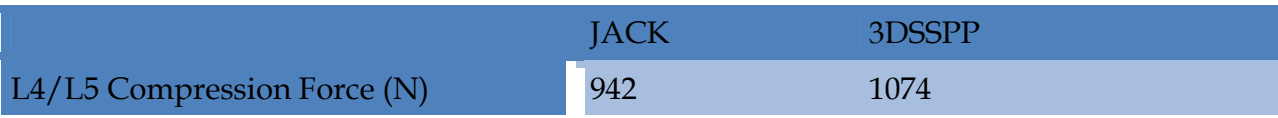

Table 23. Comparison of JACK and 3DSSPP L4/L5 Forces for Subject 3, Task 1 (See report results in Figures 43 and 47).

\section{JACK}

\section{DSSPP}

\begin{tabular}{l|}
\hline Joint \\
\hline Wrist \\
\hline Elbow \\
\hline Shoulder \\
\hline Torso \\
\hline Hip \\
\hline Knee \\
\hline Ankle
\end{tabular}

\begin{tabular}{ll}
\hline (\% Capable) & (\% Capable) \\
100 & 99 \\
100 & 100 \\
100 & 99 \\
100 & 99 \\
99 & 98 \\
100 & 99 \\
100 & 99
\end{tabular}

Table 24. Comparison of JACK and 3DSSPP Strength Capability Summary for Subject 3, Task 1. (See report results in Figures 43 and 46). 


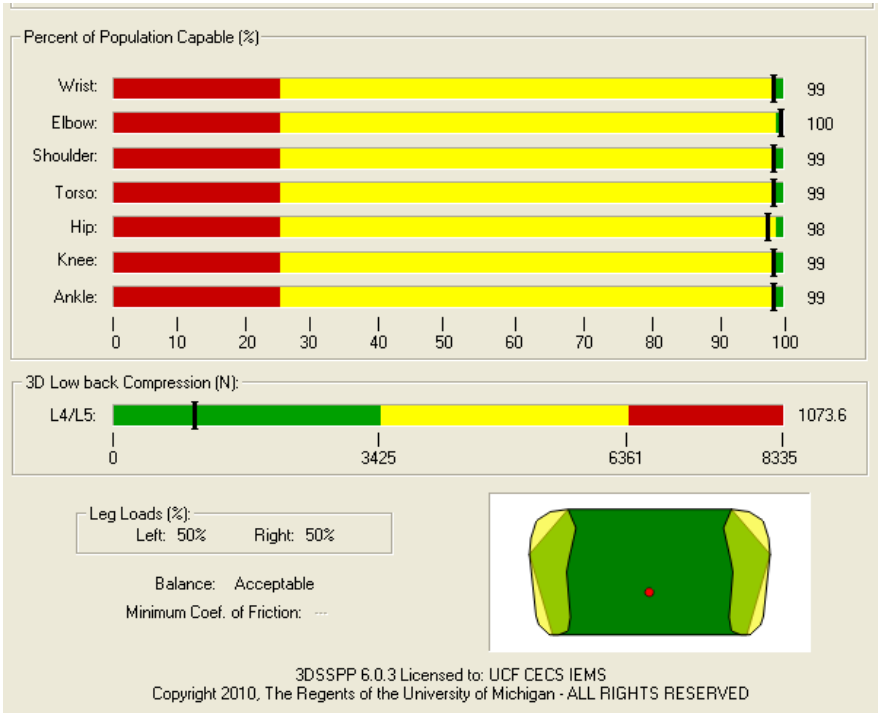

Fig. 43. 3DSSPP Summary Output, Subject 3, Task 1.
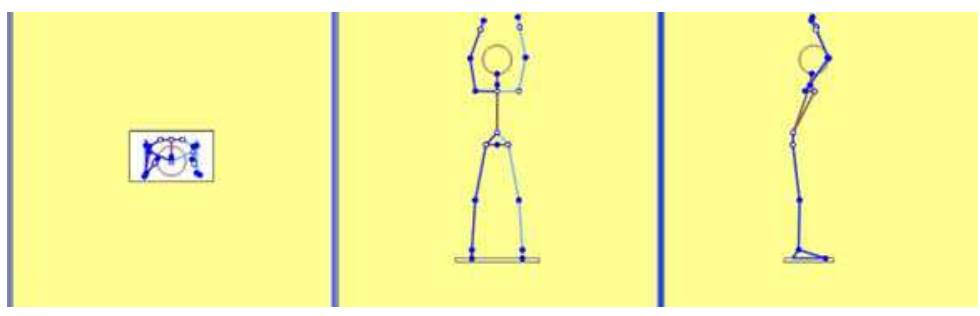

Fig. 44. 3DSSPP Simulation of Limb Angles, Subject 3, Task 1.
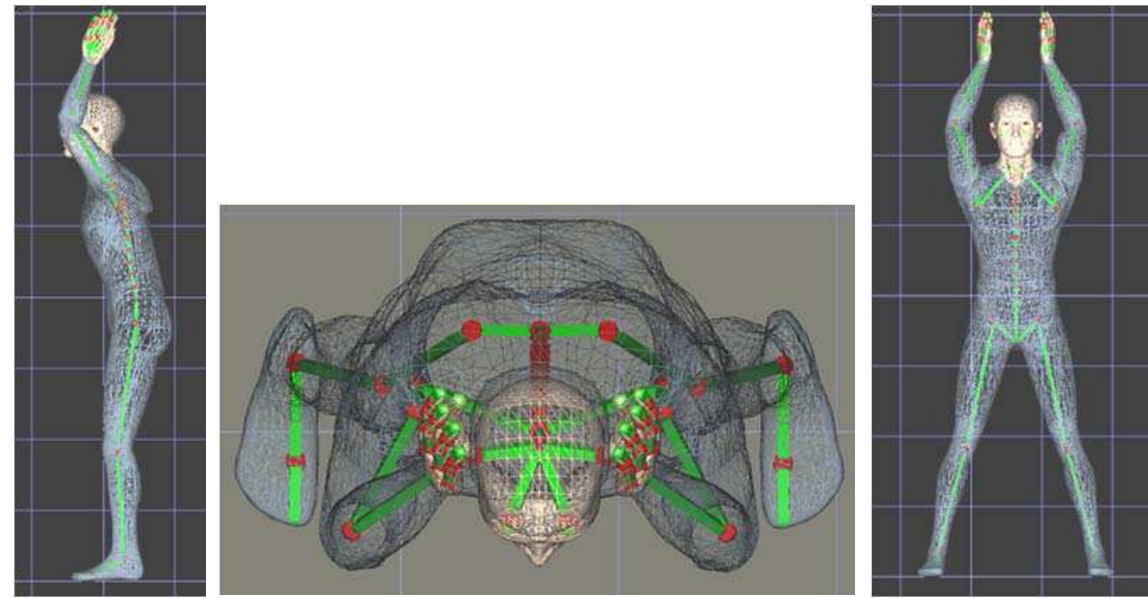

Fig. 45. JACK Simulation, Skeletal View aids in limb angle calculations. 


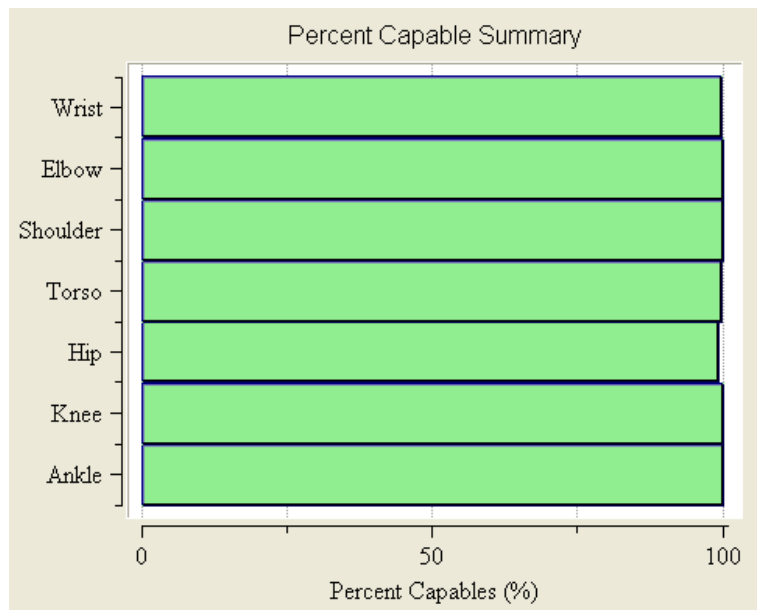

Fig. 46. JACK Output of Percent Capable, Subject 3, Task 1.

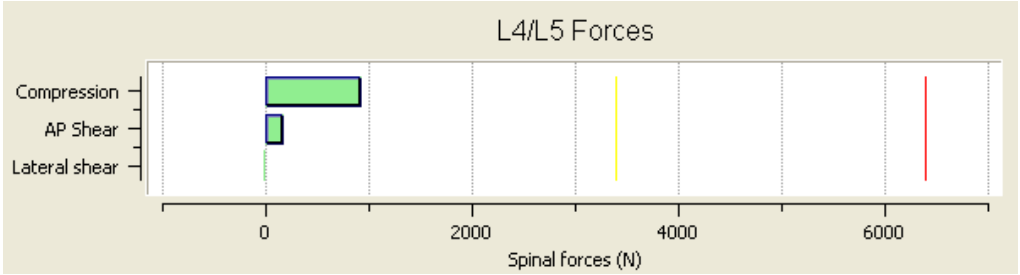

Fig. 47. JACK Output - Forces on L4/L5 for Subject 3, Task 1.

\subsection{Analysis of Subject 3 - Task 1}

For Task 2, the compression force in the lower back is below the NIOSH Back Compression Action Limit of $3400 \mathrm{~N}$. JACK reports this force is $942 \mathrm{~N}$ and 3DSSPP calculates a force of $1074 \mathrm{~N}$. Both of these values designate this task as low risk for an average person. The percent of the population capable of performing this posture ranges from 98 to 100 percent by JACK calculations and 98 to 100 percent according to 3DSSPP. According to 3DSSPP, the wrist, shoulder, torso, hip, knee and ankle area exhibit the most strain and are slightly past the NIOSH Strength Design Limit (SDL) value. JACK did not perceive any warning, as all of the joints remained in the "green" zone and were within the SDL. The lowest percentage of 98 is still generally high and may be determined a tolerable risk. However, note should be made of the yellow designation that expose joints of the body where the user population may experience some limitations in performing this task. In addition, it is important to note that although this posture is within an acceptable level according to NIOSH standards, that this posture involves holding the hands above the head with hands held behind the head. This sort of posture can lead to increased heart rate should be avoided on a repetitive or prolonged basis.

Analysis Recommendations: The low back compression force of 924.00 is below the NIOSH Back Compression Action Limit of $3400 \mathrm{~N}$, representing a nominal risk of low back injury for most healthy workers. 


\subsubsection{Subject 3, Task 2: Low-ball upper-limb save}
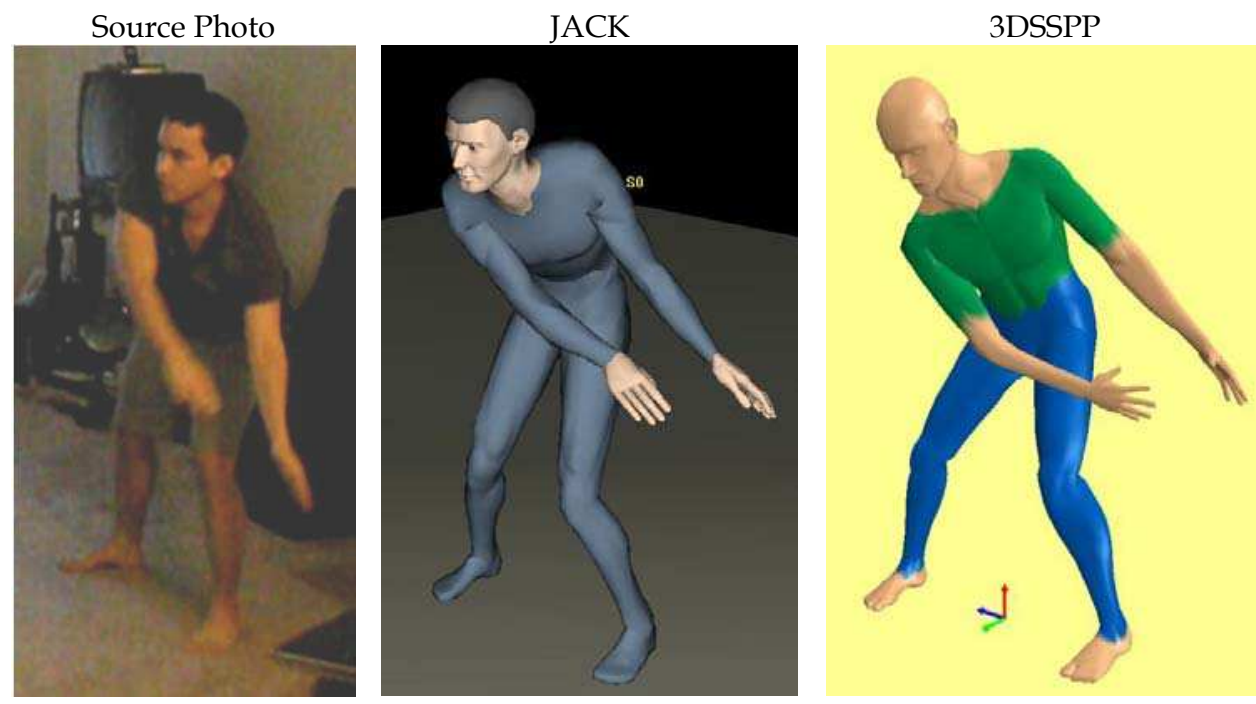

Fig. 48. Low-Ball, Upper-Limb Save; JACK; 3DSSPP.

\begin{tabular}{l|ll} 
& JACK & 3DSSPP \\
L4/L5 Compression Force $(\mathrm{N})$ & 1942 & 2071
\end{tabular}

Table 25. Comparison of JACK and 3DSSPP L4/L5 Forces for Subject 3, Task 2 (See report results in Figures 49 and 53).

\begin{tabular}{|c|c|c|}
\hline & JACK & 3DSSPP \\
\hline Joint & (\% Capable) & (\% Capable) \\
\hline Wrist & 100 & 99 \\
\hline Elbow & 100 & 100 \\
\hline Shoulder & 100 & 99 \\
\hline Torso & 99 & 98 \\
\hline Hip & 99 & \\
\hline Knee & 100 & 99 \\
\hline Ankle & 100 & 99 \\
\hline
\end{tabular}

Table 26. Comparison of JACK and 3DSSPP Strength Capability Summary for Subject 3, Task 2. (See report results in Figures 49 and 52). 


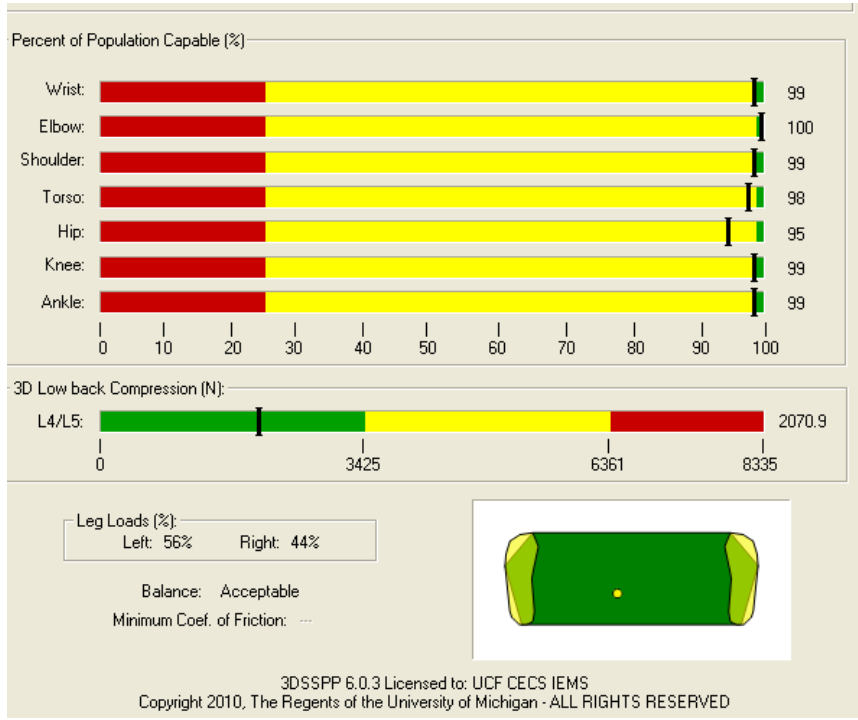

Fig. 49. 3DSSPP Summary Output, Subject 3, Task 2.
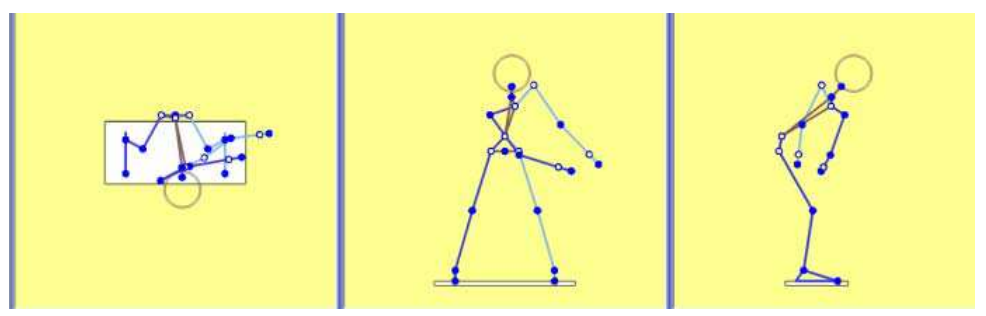

Fig. 50. 3DSSPP Simulation of Limb Angles, Subject 3, Task 2.
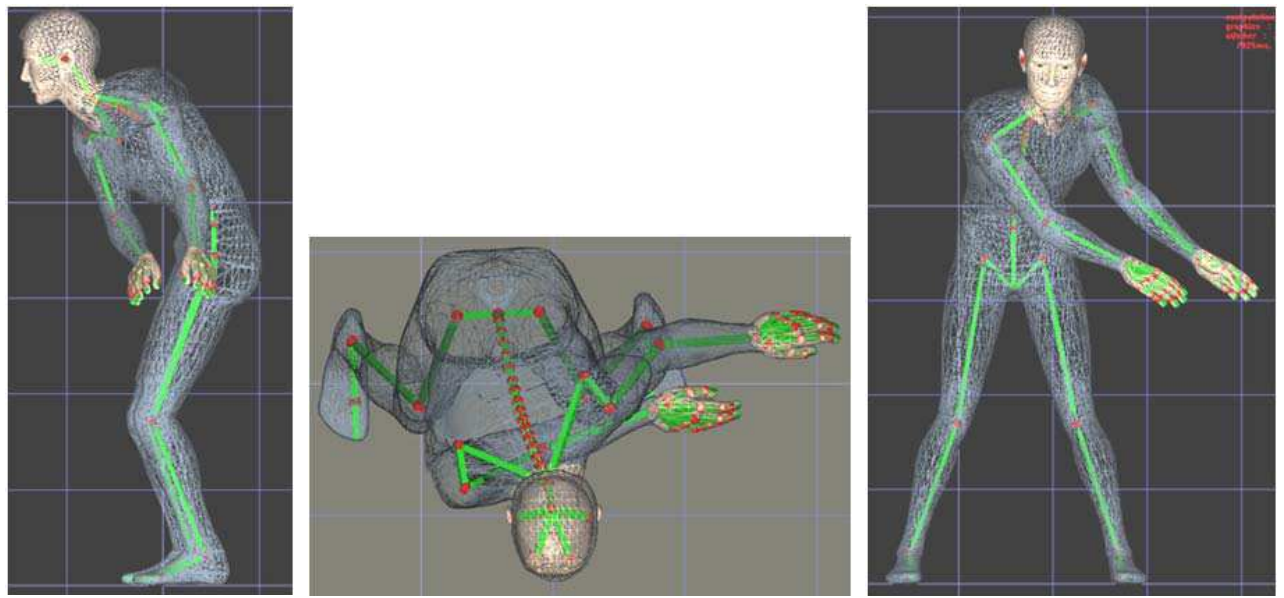

Fig. 51. JACK Simulation, Skeletal View aids in limb angle calculations. 


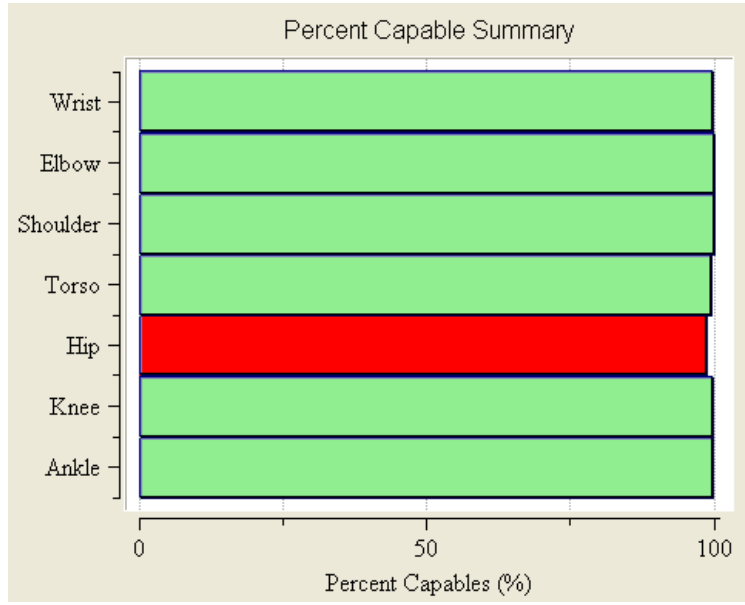

Fig. 52. JACK Output of Percent Capable, Subject 3, Task 2.

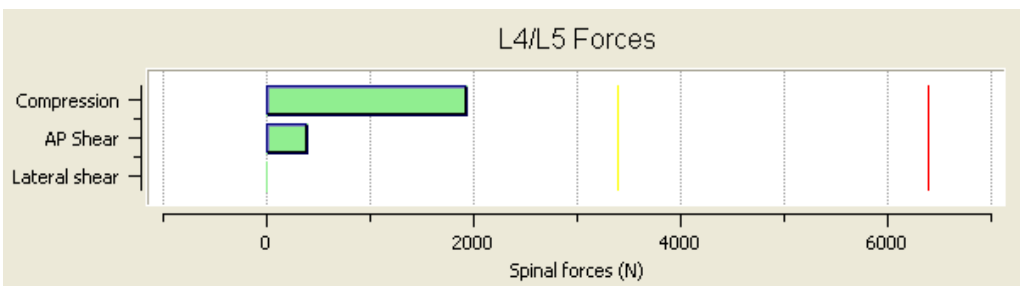

Fig. 53. JACK Output - Forces on L4/L5 for Subject 3, Task 2.

\subsection{Analysis of Subject 3 - Task 2}

For Task 3, the compression force in the lower back (L4/L5) is below the NIOSH Back Compression Action Limit of $3400 \mathrm{~N}$. JACK reports this force is $1942 \mathrm{~N}$ and 3DSSPP calculates a force of $2071 \mathrm{~N}$. Both of these values designate this task as low risk for an average person. The percent of the population capable of performing this posture ranges from 99 to 100 percent (JACK) and 95 to 100 percent (3DSSPP). According to JACK, the torso and hip area exhibit the most strain and are slightly past the NIOSH Strength Design Limit (SDL) value. 3DSSPP predicted that in addition to the torso and hip, the wrist, shoulder, knee, and ankle also exceed the action limit value. Although the lowest percentage of 95 is high and may be generally regarded as tolerable risk, the yellow designation shows the areas of the body that may limit the average person from performing this task safely. The twisting of the torso is a risky posture and reaching across the body's centerline contributes to the compression of the back, as well as the variation of load on each leg. Repetitively performing this posture may increase risk of WMSD.

Analysis Recommendations: The low back compression force of 1942.00 is below the NIOSH Back Compression Action Limit of $3400 \mathrm{~N}$, representing a nominal risk of low back injury for most healthy workers. 


\subsubsection{Subject 3, Task 3: Low-ball lower-limb save}
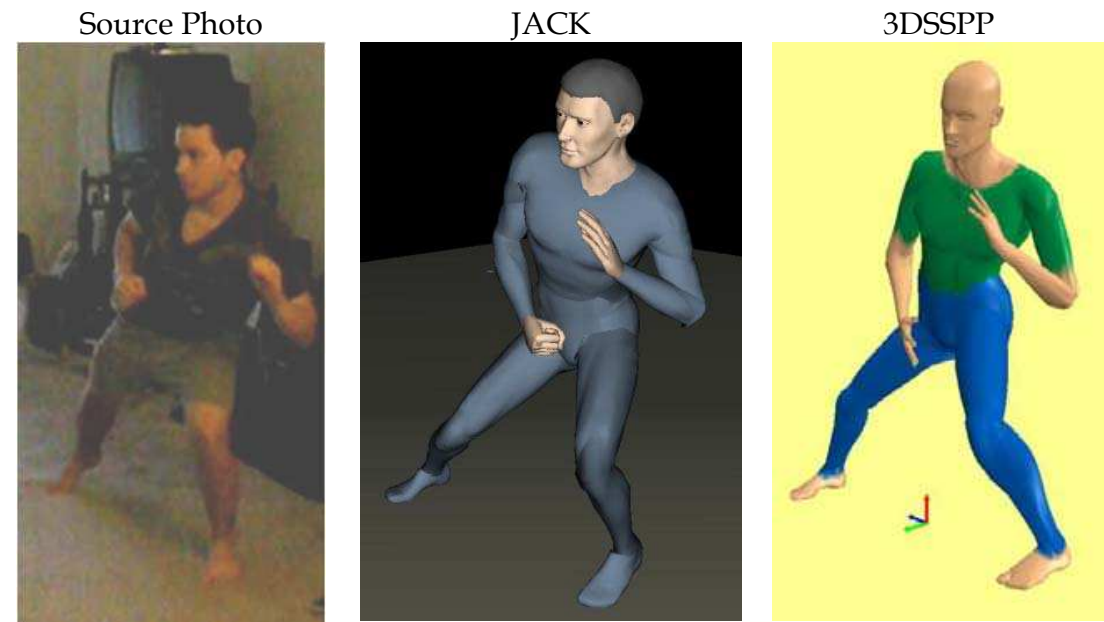

Fig. 54. Low-Ball Lower-Limb Save; JACK; 3DSSPP.

$\begin{array}{lll} & \text { JACK } & \text { 3DSSPP } \\ \text { L4/L5 Compression Force (N) } & 1656 & 1638\end{array}$

Table 27. Comparison of JACK and 3DSSPP L4/L5 Forces for Subject 3, Task 3 (See report results in Figures 55 and 59).

\begin{tabular}{l|ll} 
& JACK & 3DSSPP \\
\hline Joint & $(\%$ Capable $)$ & $(\%$ Capable $)$ \\
\hline Wrist & 100 & 99 \\
100 & 100 \\
\hline Elbow & 100 & 99 \\
\hline Shoulder & 100 & 99 \\
\hline Torso & 99 & 98 \\
\hline Hip & 79 & 98 \\
\hline Knee & 100 & 99
\end{tabular}

Table 28. Comparison of JACK and 3DSSPP Strength Capability Summary for Subject 3, Task 3. (See report results in Figures 55 and 58). 


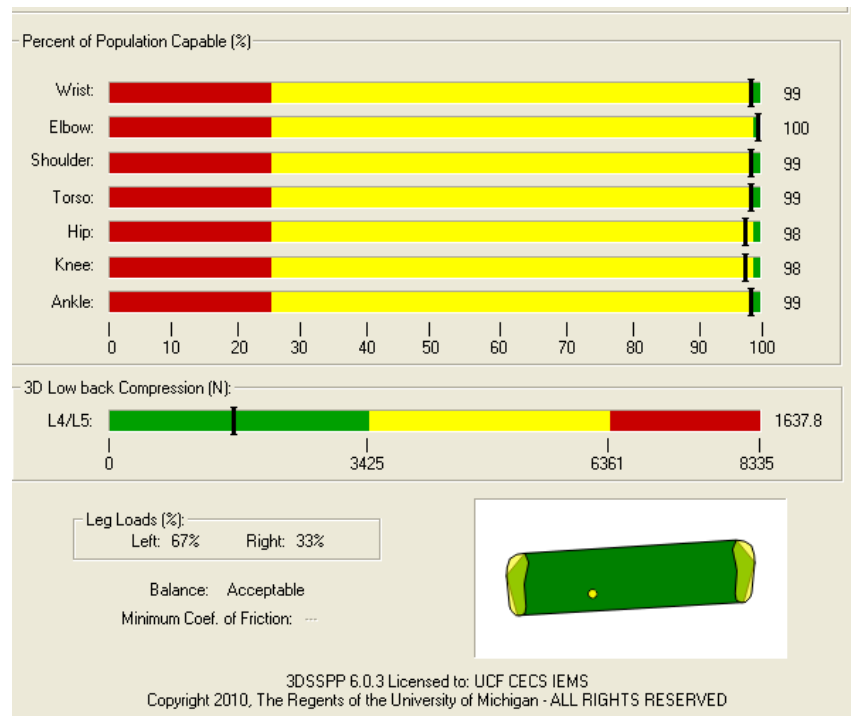

Fig. 55. 3DSSPP Summary Output, Subject 3, Task 3.

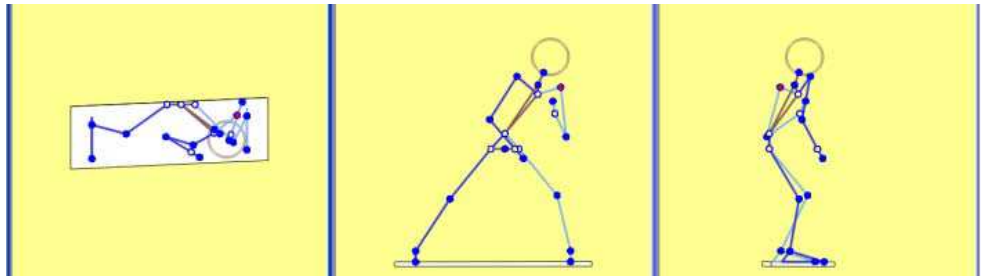

Fig. 56. 3DSSPP Simulation of Limb Angles, Subject 3, Task 3.
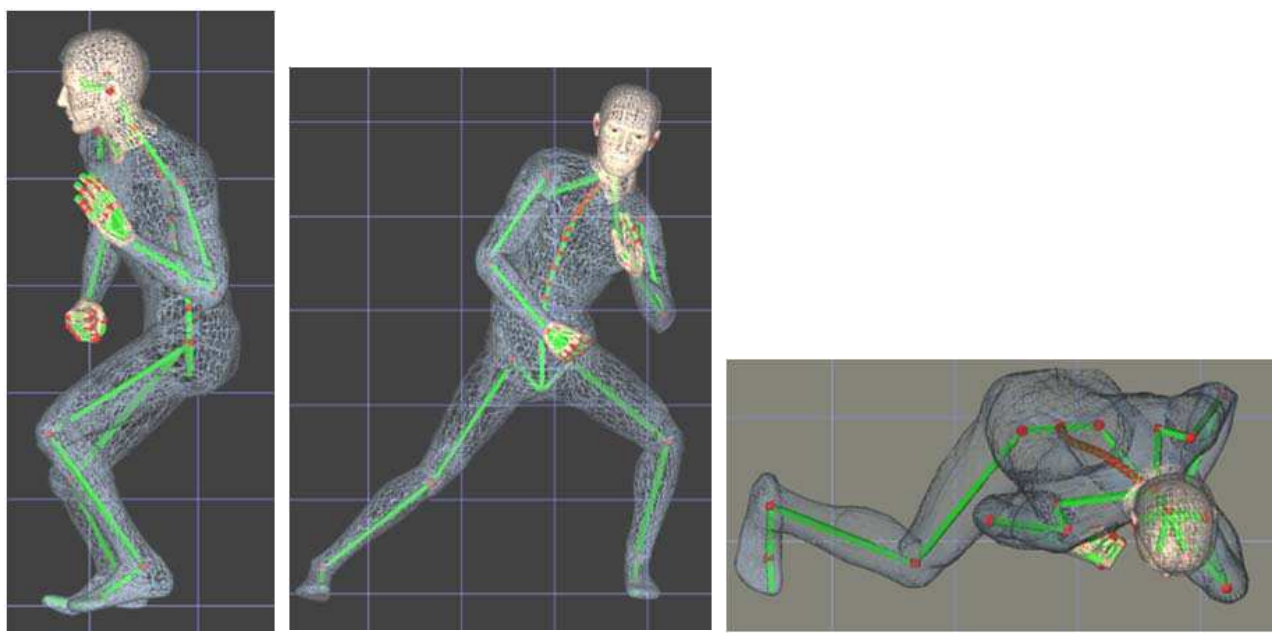

Fig. 57. JACK Simulation, Skeletal View aids in limb angle calculations. 


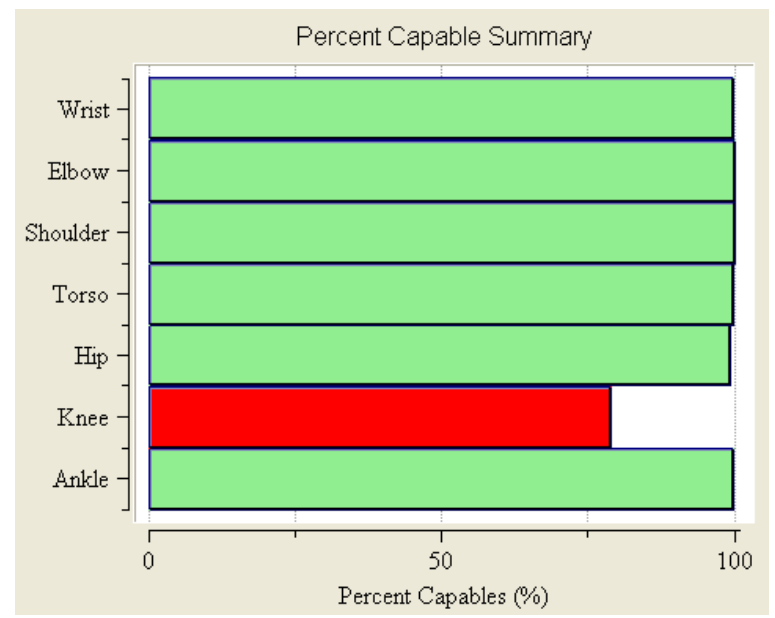

Fig. 58. JACK Output of Percent Capable, Subject 3, Task 3.

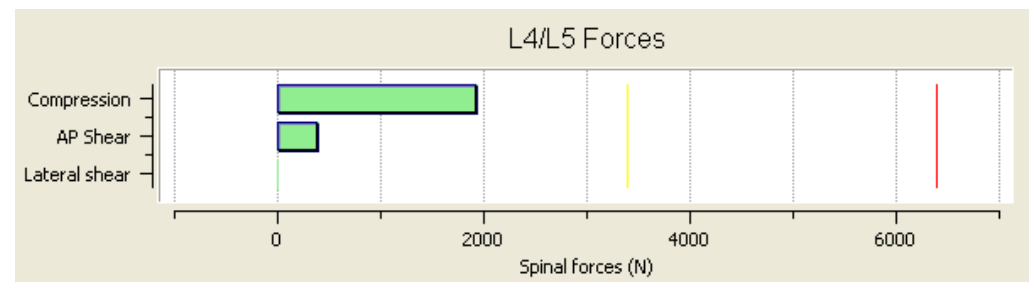

Fig. 59. JACK Output - Forces on L4/L5 for Subject 3, Task 3.

\subsection{Analysis of Subject 3 - Task 3}

For Task 4, the compression force in the lower back (L4/L5) is below the NIOSH Back Compression Action Limit of $3400 \mathrm{~N}$. JACK reports this force is $1656 \mathrm{~N}$ and 3DSSPP calculates a force of $1638 \mathrm{~N}$. Both of these values designate this task as low risk for an average person. The percentage of the population capable of performing this posture ranges from 79 to 100 percent. The hip and knee areas exhibit the most strain based on both JACK and 3DSSPP analysis. According to JACK, only $79 \%$ of the population of males can perform this posture with the load that is placed at the joint of the knee. This value is past the NIOSH design limit. This move may be very risky for users that perform this posture during game 
play on a regular basis. 3DSSPP predicted that the wrist, shoulder, knee, and ankle also exceed the action limit value. Here, the center or gravity is transferred more to the left side, which creates a variation of load on each leg. Repetitively performing this posture could lead to loss of balance and increase risk of falling or overuse injuries.

In evaluating the Kinect gaming tasks, both software analyses identify all poses as generally safe to perform. The biomechanical load on the joints may be underestimated since the jumping, rapid acceleration and deceleration of body segments, and the duration and frequency of movement are not considered in a static strength prediction. Considering these additional factors, the tasks may show different user capabilities. Thus, for the application of highly repetitive tasks, with short duration, and higher velocity of movements, these evaluation tools are limited in assessing risks. However, both programs do acknowledge this limitation as they are based on static strength predictions and employ NIOSH guidelines. Also, it is important to note that the two software tools are developed for evaluating occupational tasks. This data is useful in the preliminary assessment of the postures involved in game play, but may not be conclusive. The JACK software includes Rapid Upper Limb Assessment Tool (RULA), NIOSH, Metabolic Energy Expenditure, and Fatigue Analysis tools may be useful in further evaluating the impact of the task on the user. These features may be further explored in later experimentation.

Analysis Recommendations: The low back compression force of 1656.00 is below the NIOSH Back Compression Action Limit of $3400 \mathrm{~N}$, representing a nominal risk of low back injury for most healthy workers.

\section{Conclusion}

The intention of each of the projects included in this study is to model exact postures and retrieve biomechanical information related to selected tasks. This analysis of JACK and 3DSSPP evaluates the two software products based on the researchers' data comparison, as well as the overall user experience. The researchers found that the results of the two software packages can produce different results that sometimes lead to conflicting conclusions about the safety of a given task. For example, in the analysis of Subject 2 performing Task 1 (Pose 1) the strength capability calculated at the shoulder joint varies considerably between the packages; JACK indicated that only $15 \%$ of the population will be able to perform this pose. On the other hand, 3DSSPP predicted that $87 \%$ of population is capable of performing this pose. In this case, it is unclear as to whether this posture exceeds the NIOSH strength upper limit. It is noted that some of the variability of the results may be due to the input angles and posture manipulation controlled by the user. Differences in results may be a factor of the higher degree of freedom of joint movement that is possible by manually manipulating of the postures in 3DSSPP, which may allow the manikin to pose in a sometimes unnatural and improbable manner. JACK seems to do a better job of only allowing "realistic" human contortions. In terms of biomechanics, where force calculations are critical, these differences can present conflicting results, as observed in the results of the simulations used in this study. Despite the biomechanical conflicts, the two software packages did produce relatively similar results in the ergonomic assessment of risk associated with each task. Evaluating the software's 
assessment of tasks, based on the overall risk score, the researchers find that the packages are relatively consistent. For the lower back, the compression forces are used to evaluate the task as falling below the NIOSH Action Limit (AL) of $3400 \mathrm{~N}$ (denoted as green), between the AL and Maximum Permissible Limit of 6400 (denoted as yellow), or above the Maximum Permissible Limit (MPL) of 6400 (denoted as red). For the strength limits, the green zone is above the AL (more than $99 \%$ of healthy working population can perform the task), the yellow zone is between the AL and MPL (99\% to $25 \%$ of a healthy working population can perform the task), and the red zone is above the MPL (less than $25 \%$ of a healthy working population can perform the task). Note that when considering the "overall" score for the strength prediction, the lowest ranking joint is used as the determinant for that entire posture.

A summary of the overall ergonomic analysis from JACK and 3DSSPP can be found in table 29.

\begin{tabular}{|c|c|c|c|c|c|}
\hline & & \multicolumn{2}{|c|}{$\begin{array}{l}\text { L4/L5 Compression } \\
\text { Limit }\end{array}$} & \multicolumn{2}{|c|}{ Overall Strength Prediction } \\
\hline & & JACK & 3DSSPP & JACK & 3DSSPP \\
\hline \multirow[t]{3}{*}{ Subject 1} & Task 1 (Pose 1) & & & & \\
\hline & Task 1 (Pose 2) & & & & \\
\hline & Task 2 & & & & \\
\hline \multirow[t]{3}{*}{ Subject 2} & Task 1 & & & & \\
\hline & Task 2 & & & & \\
\hline & Task 3 & & & & \\
\hline \multirow[t]{4}{*}{ Subject 3} & Task 1 & & & & \\
\hline & Task 2 & & & & \\
\hline & Task 3 & & & & \\
\hline & Task 4 & & & & \\
\hline
\end{tabular}

Table 29. Software Comparison of Overall Ergonomic Assessment of all Tasks.

Of the 13 tasks evaluated, there was only one conflict (8\%) in the overall ergonomic lower back analysis. The comparison of JACK and 3DSSPP strength capability found conflict in 3 of the $13(23 \%)$ overall scores.

Upon evaluation of the two software packages, the researchers have made the following observations regarding the strengths and weaknesses of each product. The analysis of the software is limited to the application of the projects reported in this study, as it applies to evaluating the specific tasks aforementioned. 


\subsection{Learning curve}

JACK requires more hours dedicated to getting acquainted with its' functions and features. Completion of several tutorials was necessary to even begin using the program. The User Manual is extensive (296 pages), compared to the 3DSSPP manual. The advantage of having such a large manual is that there are several tutorials to demonstrate the use of various aspects of the program. Conversely, it is difficult to use the program without knowledge of much of the content in the manual. The index which accompanies the JACK software is not comprehensive and the users found it difficult to find solutions for encountered errors. For 3DSSPP, a review of a basic tutorial and functions from the user manual is all that is needed to begin using the software. The User Manual is more concise (122 pages) and gives explanations of analysis reports. Its index is comprehensive and one can easily locate literature on specific subjects or problem areas.

\subsection{Anthropometrics}

By Default, JACK uses ANSUR (Army Anthropometric Survey) data to scale the human models. JACK also comes standard with other databases, and it has an option that allows for user definition of individual segment lengths and weights. 3DSSPP uses National Health and Nutrition Examination Survey (NHANES) data to configure the anthropometric parameters of the human model. It is possible that some variation in the moment calculations may be due to the different data used to scale the human figure. Differences in how the two software packages defined the $X, Y$, and $Z$ coordinates and the positive and negative directions of the planes most certainly also affected the moment calculations.

\subsection{Posture manipulation and angle input}

In JACK, Human posturing techniques allow the user to quickly posture the human model while making predictions of the next movements, based on research of actual human movements and mechanics. This helped to avoid placing the manikin in an impossible posture. Joint angle manipulation was enabled for some joints (i.e., the knee), allowing the user to further manipulate the posture. Although JACK had a more intuitive inter face for posture manipulation, it was difficult to develop a system of taking angle measurements and entering them directly into the model. This meant that the majority of the posturing was created by visually referencing the photo.

3DSSPP manikin posture can be manipulated by obtaining actual angle measurements. However these measurements can be difficult to measure, unless the user has front, side and top view photos of the single pose. Another method involves using a goniometer to obtain measurements from live subjects. However, this is difficult to do while the task is being performed, as it was attempted with a live subject in the lab for the Solid Waste Collection Project. Most goniometer measurements place one arm of the goniometer along one limb, with the center at the joint and the other arm of the goniometer along the adjacent limb. This allows measurement of the angles between the two limbs. In 3DSSPP the angles requested for input are the angles taken at the joint between the limb and the horizontal. It is difficult to be sure when one is measuring a live subject that one of the 
arms is exactly at a 180 degree horizontal, often creating discrepancies in the input angles. In addition, it is also difficult to know if the user is recording the correct measurement when the subject is displaying asymmetric postures and poses that require twisting and lateral bending. Thus, even though input of angles is available, some of the limb orientations and postures of the virtual figure still have to be manually manipulated to attain the desired pose.

\subsection{Animation}

Animating the manikin in JACK with the Task Simulation Builder (TSB) is a helpful feature. This module allows the program to generate multi-step processes in a single command. For instance the "Get" command can make the human model walk over to an object, reach, and pick it up, in a single command. This feature is useful for evaluating manufacturing processes and multi-step tasks. In addition to the human animation, 3D objects can be incorporated into the scene to represent components of the environment, object being handled (i.e. materials, tools), and workstations/machinery. These objects can also be animated. Postures or tasks are input at specified time intervals or frames and the program predicts the iterations of poses and actions required to perform the task. One issue encountered with the TSB is that the software does not allow a human model to be the load, as in a victim being lifted (disaster research). However, the program does allow for multiple humans and objects to be involved in the animation. Another weakness encountered was a shift in the frame of reference for the object being lifted and inexplicable changes to the position of the hands grasping the object. This hand shifting error occurred when a manikin was selected which differed from the manikin representing the 50th percentile human. Another frequent error occurred in which the object seemed to float at odd angles through the simulation, even if the virtual human was grasping it in a pre-defined spot. The manual did not offer insight into how this problem could be corrected. It obviously was related to the matrix used as the frame of reference and the center of mass for the object; however, multiple iterations of various simulations encountered this problem.

3DSSPP also allows for animation; however, it is much more limited. The user can input postures at specified frames and the software will do some limited prediction of movement from one posture to the next. The software has some simple objects such as a box that can be scaled and placed only in the manikin's hand. 3DSSPP does not allow for multiple people to perform the tasks simultaneously, as is often the case in real-time occupational settings. Also, the "dynamic" simulation feature essentially just compiles a series data for each pose as a static load.

Overall, the information and analyses provided by JACK and 3DSSPP can be used to aid in evaluating physically-intensive tasks, redesigning a task, designing products, and evaluating the ergonomic impact on a worker or user. For general occupational simulations, such as workstation design and workload distribution, exact angles aren't needed for individual poses. Simple lifting calculations can provide extremely useful ergonomic design and consideration for most occupational applications. However, where exact posture replication is desired, the user may have to employ supplemental or alternative technologies such as 3D Motion Capture. 


\subsection{Limitations}

In the attempt to replicate unique and awkward postures, it is apparent that obtaining and applying the joint angles is an important factor in the output of the forces, moments, and strength prediction. When manipulating the manikin in 3DSSPP, the researchers noticed how a single degree in rotation of the wrist or shoulder can render the posture safe or unsafe. This fact supports the hypothesis that the variability of results between the forces and moments output may very well be due to inaccurate replication of the posture and angles, even if the postures appeared to be the same visually. Another limitation of the study is that only one camera was available, and the subject could not be simultaneously recorded from the front, side, and top view. If images from multiple planes of the same posture were obtained, then segment angles would be easier to find and replicate. This would have allowed for more accurate angle measurements. The JACK software is enabled with a Motion Capture Module that allows direct input of 3D motion data of an actual human subject. This requires expensive hardware but can give the body segment angles that are hard to manually measure. This may help to correct the human error in manually entering the angle measurements and arbitrarily manipulating the manikin's posture. One other major limitation of this study is that static postures were evaluated and not the dynamic movements of the subject. The biomechanical load on the joints may be underestimated by this limitation. Jumping, rapid acceleration and deceleration of body segments, and the duration and frequency of movement may also yield different analysis of risks and would be a necessary study for a complete comparison of the software. Dynamic Biomechanical Analysis is not an available feature of either JACK or 3DSSPP. Although JACK does allow for complex animation, it does not account for the effects of acceleration and momentum. The "dynamic" reports generated by JACK are essentially the data collected at a fixed moment in time, as in 3DSSPP, the reports are basically a series of static evaluations.

"Static Strength Prediction (SPP) is most useful for analyzing tasks that involve slow movements, since the calculations assume that the effects of acceleration and momentum are negligible." (JACK Training Manual, p. 18)

This poses an issue, especially with the Interactive Gaming study. If the program could account for speed and frequency of the motions, then a more thorough biomechanical analysis of gameplay could be observed. The RULA, NIOSH, Metabolic Energy Expenditure, and Fatigue Analysis tools provided in the JACK program could prove to give better insight into the ergonomic risk of the task, but will not provide biomechanical data.

\subsection{Future areas of related research}

Many software packages claim to perform biomechanical analysis of user-input data. The majority of these packages are used in sports analysis. A large percentage of the software requires specialized hardware, often requiring and expensive investment. The ideal software for use in research has yet to be identified, and may not currently exist. Features of an optimal software package would allow upload of user-supplied photos and video. The ultimate usability feature would be to allow upload of footage, including from news 
footage, for analysis of tasks. The ability to simulate and analyze the movements of multiple subjects simultaneously would find frequent application, especially in the disaster management realm. Graphical representation of the results, similar to the 3DSSPP output is useful to quickly identify tasks which place subjects at risk. While creating simulations, usability would be enhanced if software prompted user with suggestions to correct errors. Interactive user guides which focus on common errors and steps to correct encountered errors would be of great use to researchers and facilitate simulations. Other suggestions for further research may include exploring whether the analysis module in JACK (NIOSH, RULA, etc.) can directly use the animation data to automatically calculate output, rather than have the user manually enter the frequency, cycle time, lifting height, etc. Additionally, a Usability Study comparing the Human Modeling software may be an appropriate research topic to further expand on this study. The researchers in this study found many limitations with regard to data input and errors, as previously discussed. The learning curve for both software packages is extensive. Enhancements to the training manuals and interactive features would greatly improve the usability of both software packages and allow for a comprehensive comparative evaluation.

\section{References}

All Sport Systems (n.d). MotionView Video Analysis Software, In: All Sport Systems, 15 February 2011, Available from: < http://www.allsportsystems.com/>

Almedghio, S. A. (2009). Wii knee revisited: meniscal injury from 10-pin bowling, In: BMJ Case Reports, 29 July 2011, Available from:

<http://casereports.bmj.com/content/2009/bcr.11.2008.1189.full>

AnyBody Technology Inc. (n.d.). AnyBody Tech Modeling Systems, In: AnyBody Technology Inc., 8 August 2011, Available from:

<http:/ / www.anybodytech.com/index.php?id=26>Barron, D. A. (2008). Wii Knee. Emergency Radiology, Vol. 15, No.4, pp. (255-257)

C-Motion, Inc. (2010). C-Motion Research Biomechanics, In: C-Motion, Inc., 15 February 2011, Available from: <http://www.c-motion.com/ products/visual3d.php>

Collins, M. N. (2008). Magnetic resonance imaging of acute 'wiitis' of the upper extremity. Skeletal Radiology, Vol.37, No.5, pp. (481-3)

Das, A. (April 20 2009). More Wii Warriors Are Playing Hurt, In: NY Times, 29 July 2011, Available from:

<http:/ / www.nytimes.com/2009/04/21/health/21wii.html?adxnnl=1\&partner=rs s\&emc $=$ rss\&adx nnlx=1302683371-q/diaU3ZZNGogDlxOaFobg $>$

Georgia Tech Research Institute (2011). Occupational Safety and Health Program, In: Georgia Tech Research Institute, 15 June 2011, Available from:

<http://www.oshainfo.gatech.edu/index.html>

Innovision Systems INC. (n.d). MaxPRO, In: Innovision Systems INC. February 27, 2011, Available from: <http://www.innovision- systems.com/>

Jacobs, A. (2008). A Rescue in China, Uncensored, In: The New York Times, 15 June 2011, Available from:

<http://www.nytimes.com/2008/05/14/world/asia/14response.html> 
Kano, M., Sigel, JM., \& Bourque, LB. (2005). First-aid training and capabilities of the lay public: A potential alternative source of emergency medical assistance following a natural disaster. Disasters, Vol. 29, No.1, PP. (58-74)

LA County SAR (2010). Haiti Earthquake, In: LA County SAR, 15 June 2011, Available from: <http:/ / edwardrees.wordpress.com/2010/01/21/3-days/>

Laursen, B., Schibye, B. (2002). The effect of different sources on biomechanical loading of shoulder and lumbar spine during pushing and pulling of two-wheeled containers. Applied Ergonomics, Vol. 33, No. 2, pp. (167-174).

McNeil, S. (1999). An exploration of the Opportunities, Costs, Benefits, and issues Related to Automation of Solid Waste Collection Vehicles. Environment Research \& Education Foundation.

Musculographics Inc. (2011). 15 February 2011, Available from: http://www.musculographics.com/

National Climatic Data Center, "Billion Dollar U.S. Weather Disasters". http://www/ncdc.noaa.gov/oa/reports/billionz.html, retrieved December 13, 2010.

New Jersey National Guard (n.d). Hurricane Katrina, In: New Jersey National Guard, 15 June 2011, Available from: <http://www.pdcbank.stat.nj.uc/military/publication/guardlife/volume31no5>

Siemens PLM Software (2011). JACK, In: Siemens PLM Software, 15 July 2011, Available from: <http://www.plm.automation.siemens.com/en_in/Images/4917_tcm6414952.pdf $>$

Siemens PLM Software (2011). Lowback Analysis Compression Tool Background, In: Siemens PLM Software, 25 July 2011, Available from: <C: $\backslash$ Program Files $\backslash$ Siemens $\backslash$ Jack_7.0\library $\backslash$ help $\backslash$ TAT_Low_back.htm>

Siemens PLM Software (2011). Task Analysis Toolkit (Tat) Training Manual, In: Siemens PLM Software, 25 July 2011, Available from:

<http://www.plm.automation.siemens.com/en_us/products/tecnomatix/assemb ly_planning/jack/index.shtml>

Siemens PLM Software (2011). Static Strength Prediction Tool Background, In: Siemens PLM Software, 26 July 2011, Available from:

<file:///C:/Program\%20Files/Siemens/Jack_7.0/library/help/TAT_Strength.htm \#top>

Stull, J. (March, 2006), Understanding PPE selection \& use during disasters. Professional Safety, Vol. 51, No.3, pp. (18-49)

University of Michigan (2010). 3D Static Strength Prediction Program, In: University of Michigan, February 27, 2011, Available from:

<http://www.engin.umich.edu/dept/ioe/3DSSPP/>

University of Waterloo (n.d.). Ergowatch, In: Ergonomics and Safety Consulting Services, 8 August 2011, Available from: <http:/ / www.escs.uwaterloo.ca/brochure.pdf >

Xcitex, Inc (2009). ProAnalyst Software, In: Xcitex, Inc, 15 February 2011, Available from: <http://www.xcitex.com/html/proanalyst_applications_examples.php> 
Youngstown State University, Environmental and Occupational Health and Safety (1997). Back Belts Pros and Cons, In: Youngstown State University, 15 June 2011, Available from: <http://cc.ysu.edu/eohs/bulletins/Lifting\%20Belts.htm> 


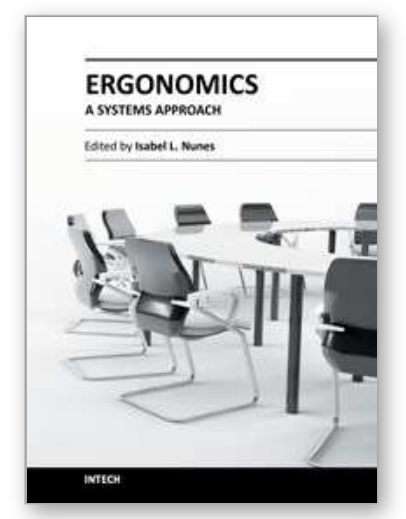

\author{
Ergonomics - A Systems Approach \\ Edited by Dr. Isabel L. Nunes
}

ISBN 978-953-51-0601-2

Hard cover, 232 pages

Publisher InTech

Published online 25, April, 2012

Published in print edition April, 2012

This book covers multiple topics of Ergonomics following a systems approach, analysing the relationships between workers and their work environment from different but complementary standpoints. The chapters focused on Physical Ergonomics address the topics upper and lower limbs as well as low back musculoskeletal disorders and some methodologies and tools that can be used to tackle them. The organizational aspects of work are the subject of a chapter that discusses how dynamic, flexible and reconfigurable assembly systems can adequately respond to changes in the market. The chapters focused on Human-Computer Interaction discuss the topics of Usability, User-Centred Design and User Experience Design presenting framework concepts for the usability engineering life cycle aiming to improve the user-system interaction, for instance of automated control systems. Cognitive Ergonomics is addressed in the book discussing the critical thinking skills and how people engage in cognitive work.

\title{
How to reference
}

In order to correctly reference this scholarly work, feel free to copy and paste the following:

Pamela McCauley Bush, Susan Gaines, Fatina Gammoh and Shanon Wooden (2012). A Comparison of Software Tools for Occupational Biomechanics and Ergonomic Research, Ergonomics - A Systems Approach, Dr. Isabel L. Nunes (Ed.), ISBN: 978-953-51-0601-2, InTech, Available from:

http://www.intechopen.com/books/ergonomics-a-systems-approach/a-comparison-of-software-tools-foroccupational-biomechanics-and-ergonomics-research

\section{INTECH}

open science | open minds

\section{InTech Europe}

University Campus STeP Ri

Slavka Krautzeka 83/A

51000 Rijeka, Croatia

Phone: +385 (51) 770447

Fax: +385 (51) 686166

www.intechopen.com

\section{InTech China}

Unit 405, Office Block, Hotel Equatorial Shanghai

No.65, Yan An Road (West), Shanghai, 200040, China

中国上海市延安西路65号上海国际贵都大饭店办公楼 405 单元

Phone: +86-21-62489820

Fax: $+86-21-62489821$ 
(C) 2012 The Author(s). Licensee IntechOpen. This is an open access article distributed under the terms of the Creative Commons Attribution 3.0 License, which permits unrestricted use, distribution, and reproduction in any medium, provided the original work is properly cited. 